\title{
Features of Bifurcations of Periodic Solutions of the Ikeda Equation
}

\author{
E.P. Kubyshkin, A. R. Moriakova
}

We study equilibrium states and bifurcations of periodic solutions from the equilibrium state of the Ikeda delay-differential equation well known in nonlinear optics. This equation was proposed as a mathematical model of a passive optical resonator in a nonlinear environment. The equation, written in a characteristic time scale, contains a small parameter at the derivative, which makes it singular. It is shown that the behavior of solutions of the equation with initial conditions from the fixed neighborhood of the equilibrium state in the phase space of the equation is described by a countable system of nonlinear ordinary differential equations. This system of equations has a minimal structure and is called the normal form of the equation in the neighborhood of the equilibrium state. The system of equations allows us to pick out one "fast" and a countable number of "slow" variables and apply the averaging method to the system obtained. It is shown that the equilibrium states of a system of averaged equations with "slow" variables correspond to periodic solutions of the same type of stability. The possibility of simultaneous bifurcation of a large number of stable periodic solutions(multistability bifurcation) is shown. With further increase in the bifurcation parameter each of the periodic solutions becomes a chaotic attractor through a series of period-doubling bifurcations. Thus, the behavior of the solutions of the Ikeda equation is characterized by chaotic multistability.

Keywords: Ikeda equation, periodic solutions, bifurcation of multistability, chaotic multistability

Received July 19, 2018

Accepted August 29, 2018

Evgenii P. Kubyshkin

kubysh.e@yandex.ru

Alena R. Moriakova

alyona_moryakova@mail.ru

P. G. Demidov Yaroslavl State University

ul. Sovetskaya 14, Yaroslavl, 150003, Russia

RUSSIAN JOURNAL OF NONLINEAR DYNAMICS, 2018, 14(3), 301-324 


\section{Introduction}

We consider the following delay-differential equation

$$
\dot{x}=\mu \sin (x(t-\tau)-c)-x .
$$

This equation was proposed in [1] to describe the dynamics of a passive optical resonator. In (1.1) the variable $x(t)$ determines the phase shift of the electric field in the nonlinear medium of a ring resonator, $\tau$ is the light propagation time in the ring resonator $0 \leqslant c<2 \pi-$ a constant phase shift, and $\mu>0$ is the coefficient characterizing the intensity of the laser emission. Equation (1.1), called the Ikeda equation, is widely used in nonlinear optics to model nonlinear optical effects. Solutions of the Ikeda equation have been studied in [1-4], where the multiplicity of equilibrium states was shown and the existence of a variety of periodic solutions and chaotic oscillations was shown using numerical integration. In [5], the problem of reconstructing parameters of the equation from the observed oscillations is considered. In [6], an information transmission line with a chaotic carrier was proposed and studied using the Ikeda equation.

Depending on the values of the parameters $c$ and $\mu$, Eq. (1.1) can have a large number of different stable and unstable equilibrium states. Some equilibrium states can arise in stableunstable pairs. The loss of stability of an equilibrium state with changing parameters leads to a bifurcation of a certain number (depending on the behavior of the parameters) of stable (and unstable) periodic solutions, i.e., there is a bifurcation of multistability.

With further changes in the bifurcation parameters, each such periodic solution becomes a chaotic attractor through a series of period-doubling bifurcations. The dynamics of the solutions of the equation exhibits chaotic multistability. This paper is devoted to the study of these problems. The results obtained provide insight into the structure of the phase space of the equation. The method of uniform normalization, proposed in [7], is used as the research method. Some particular results in the study of Eq. (1.1) are outlined in the authors' note [8].

\section{Mathematical formulation of the problem, analysis of equilibrium states}

In real optical systems the delay $\tau$ is substantially larger than the characteristic time of electric field oscillations. Passing to the dimensionless time $t^{\prime}=t / \tau$ in Eq. (1.1) and setting $\varepsilon_{1}=\tau^{-1} \ll 1$, we get the equation

$$
\varepsilon_{1} \dot{x}(t)+x(t)=\mu \sin (x(t-1)-c),
$$

where $\varepsilon_{1}$ is a small parameter.

The equilibrium states $x_{*}(c, \mu)$ of Eq. (2.1) are determined by the roots of the equation

$$
x=\mu \sin (x-c)
$$

depending on $c$ and $\mu$. We describe the equilibrium states and investigate their stability. The stability of $x_{*}(c, \mu)$ is determined by the location of the roots of the characteristic equation

$$
P\left(\lambda ; \varepsilon_{1}\right) \equiv \varepsilon_{1} \lambda+1-\mu \cos \left(x_{*}(\mu, c)-c\right) \exp (-\lambda)=0 .
$$

For $\left|\mu \cos \left(x_{*}(\mu, c)-c\right)\right|<1$ and $0<\varepsilon_{1} \leqslant \varepsilon_{0}$, where $\varepsilon_{0}$ is small, all roots of Eq. (2.3) lie in the left complex half-plane. In this case the equilibrium state $x_{*}(c, \mu)$ is asymptotically stable. 
For $\left|\mu \cos \left(x_{*}(\mu, c)-c\right)\right|>1$ and $0<\varepsilon_{1} \leqslant \varepsilon_{0}$ Eq. (2.3) has roots lying in the right complex half-plane, i.e., the equilibrium state $x_{*}(c, \mu)$ is unstable [9]. The case $\left|\mu \cos \left(x_{0}(\mu, c)-c\right)\right|=1$ is a boundary case. This equality defines in the plane $c, \mu$ the set of bifurcation points of periodic solutions from the equilibrium state $x_{*}(c, \mu)$.

For $0<c<2 \pi$ Eq. (2.2) has a single solution $x_{*}(\mu, c), \lim _{\mu \rightarrow 0} x_{*}(\mu, c)=0$, where $-\pi<x_{*}(\mu, c)<0$ if $0<c<\pi$ and $x_{*}(\mu, \pi) \equiv 0,0<x_{*}(\mu, c)<\pi$ if $\pi<c<2 \pi$. For $c=0$ Eq. (2.2) has the solution $x_{*}(\mu, c) \equiv 0$, and for $\mu>1$ two solutions $x_{*}(\mu, c)>0$ $\left(\lim _{\mu \rightarrow 1} x_{*}(\mu, c)=0\right)$ and $-x_{*}(\mu, c)$.

Also, for every $c$ there is a sequence $0<\mu_{1}(c) \leqslant \mu_{2}(c) \leqslant \ldots$ for which Eq. (2.2) has multiple roots $x^{-}{ }_{*}\left(\mu_{k}(c), c\right)=x^{+}{ }_{*}\left(\mu_{k}(c), c\right), \mu_{k}(c) \cos \left(x_{*}^{ \pm}\left(\mu_{k}(c), c\right)\right)=1$, which correspond to paired (stable and unstable) equilibrium states $x^{-}{ }_{*}(\mu, c)$ and $x^{+}{ }_{*}(\mu, c)$ of Eq. (2.1) for $\mu>\mu_{k}(c)$. With further increase in the parameter $\mu$ the equilibrium state $x^{-} *(\mu, c)$ loses stability for some $\mu_{*}$ and $\mu_{*} \cos \left(x_{*}^{-}\left(\mu_{*}, c\right)\right)=-1$.

Figure 1 shows diagrams of equilibrium states of Eq. (2.1) for different values of $c$ and $0 \leqslant \mu \leqslant 10$. Stable equilibrium states are marked by thick lines, unstable ones, by thin lines. Equilibrium states $x_{*}\left(\mu_{*}(c), c\right)$ losing stability under the conditions $\mu_{*}(c) \cos \left(x_{*}\left(\mu_{*}(c), c\right)\right)=1$ and $\mu_{*}(c) \cos \left(x_{*}\left(\mu_{*}(c), c\right)\right)=-1$ are shown as dark and light circles, respectively. Further increase in the parameter $\mu$ does not change the picture of the equilibrium states of Eq. (2.1) a new pair (stable and unstable) of equilibrium states $x^{-}(\mu, c)$ and $x^{+}{ }_{*}\left(\mu_{k}(c), c\right)$ appears, the first of which later loses stability at some $\mu_{*}$ with $\mu_{*} \cos \left(x_{*}^{-}\left(\mu_{*}, c\right)\right)=-1$.

There are three types of stability loss of equilibrium states in Eq. (2.1): when the equilibrium state $x_{*}(\mu, c)$ loses stability for $\mu_{*}$ with $\mu_{*} \cos \left(x_{*}^{-}\left(\mu_{*}, c\right)\right)=-1$, when at $c=0$ the equilibrium state $x_{*}(\mu, 0) \equiv 0$ at the point $\mu_{*}=1$ loses stability for $\mu_{*} \cos \left(x_{*}^{-}\left(\mu_{*}, 0\right)\right)=1$, and at the point of appearance of the multiple root of Eq. (2.2) after the birth of paired equilibrium states $x^{-} *(\mu, c)$ and $x^{+}(\mu, c)$.

We consider the first case of stability loss as the most frequent for the equilibrium state $x_{*}(\mu, c)(0<c<2 \pi), \lim _{\mu \rightarrow 0} x_{*}(\mu, c)=0$.

We perform in Eq. (2.1) the substitution

$$
x(t)=x_{*}(\mu, c)+y(t)
$$

and represent the nonlinear part of Eq. (2.1) with a Taylor series. As a result, in a neighborhood of the equilibrium state $x_{*}(\mu, c)$ the behavior of the solutions of Eq. (2.1) is determined by the following equation:

$$
\varepsilon_{1} \dot{y}(t)+y(t)-\mu \cos \left(x_{*}(\mu, c)-c\right) y(t-1)+f(y(t-1) ; \mu, c)=0,
$$

where $f(y ; \mu, c)=b_{2}(\mu, c) y^{2}+b_{3}(\mu, c) y^{3}+o\left(y^{3}\right)$ is an analytic function in a neighborhood of $y=0$, where

$$
b_{2}(\mu, c)=x_{*}(\mu, c) / 2, b_{3}(\mu, c)=\mu \cos \left(x_{*}(\mu, c)-c\right) / 6 .
$$

We define the function $\mu=\mu_{*}(c)$ from the condition $\mu_{*}(c) \cos \left(x_{*}\left(\mu_{*}(c), c\right)-c\right) \equiv-1$. The graph of the function $\mu_{*}(c)$ is shown in Fig. 2.

Assuming that $c=c_{*}$ is fixed, from the equation

$$
\left(\mu_{*}\left(c_{*}\right)+\mu\right) \cos \left(x_{*}\left(\mu_{*}\left(c_{*}\right)+\mu, c_{*}\right)-c_{*}\right)=-1-\varepsilon_{2},\left|\varepsilon_{2}\right| \ll 1,
$$

with $\left.\left(\left(\mu_{*}(c)+\mu\right) \cos \left(x_{*}\left(\mu_{*}(c)+\mu, c\right)-c\right)\right)_{\mu}\right|_{\mu=0}=-\left(1+x_{*}\left(\mu_{*}(c), c\right)^{2} /\left(2 \mu_{*}(c)\right)=\mu_{1}^{-1}(c)>0\right.$, we get $\mu=\mu\left(\varepsilon_{2}\right)$. Hence, we have

$$
\mu=\mu_{*}\left(c_{*}\right)+\mu\left(\varepsilon_{2}\right)=\mu_{*}\left(c_{*}\right)+\mu_{1}\left(c_{*}\right) \varepsilon_{2}+o\left(\varepsilon_{2}\right) .
$$



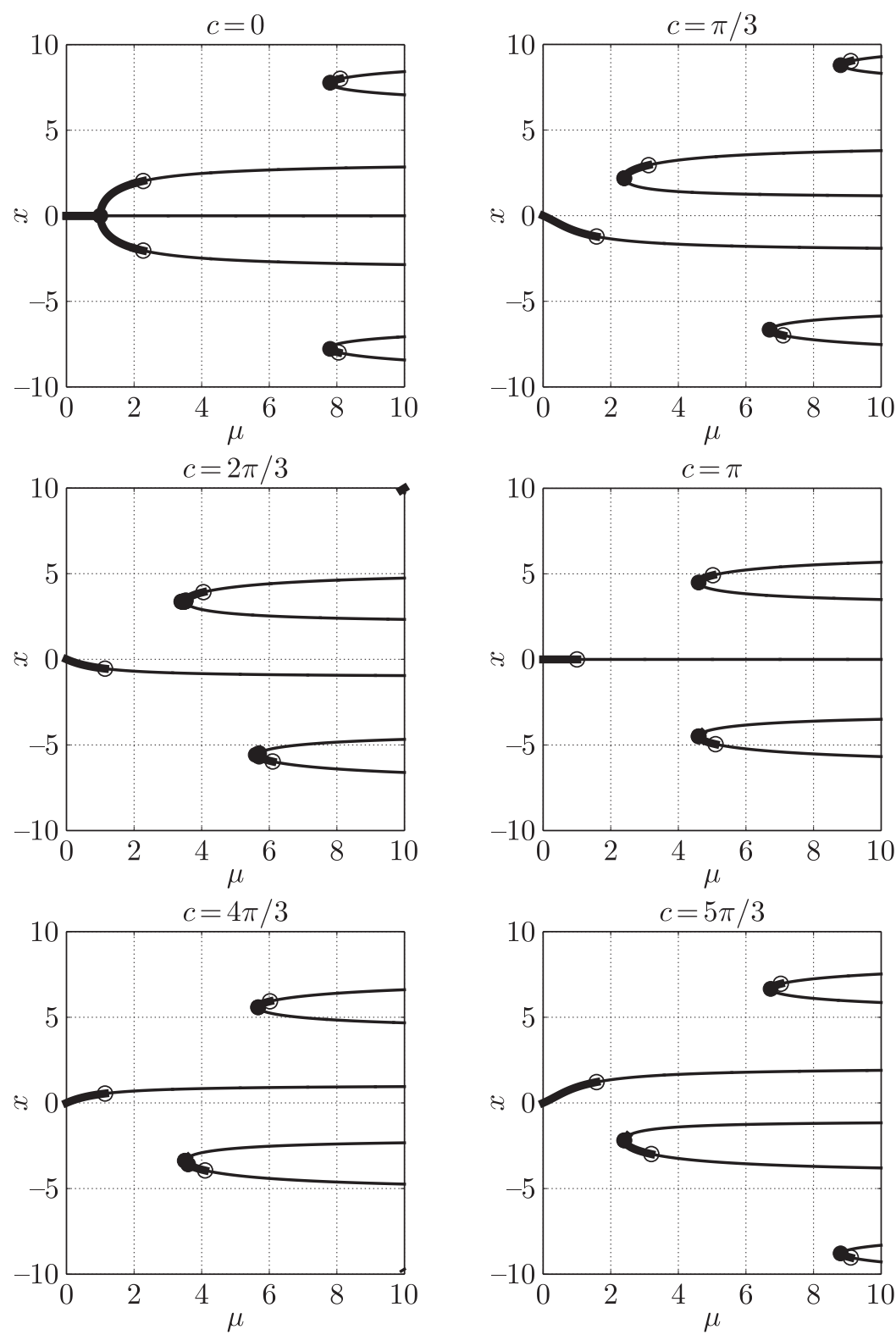

Fig. 1. Equilibrium states of Eq. (1.1) depending on parameter $c$.

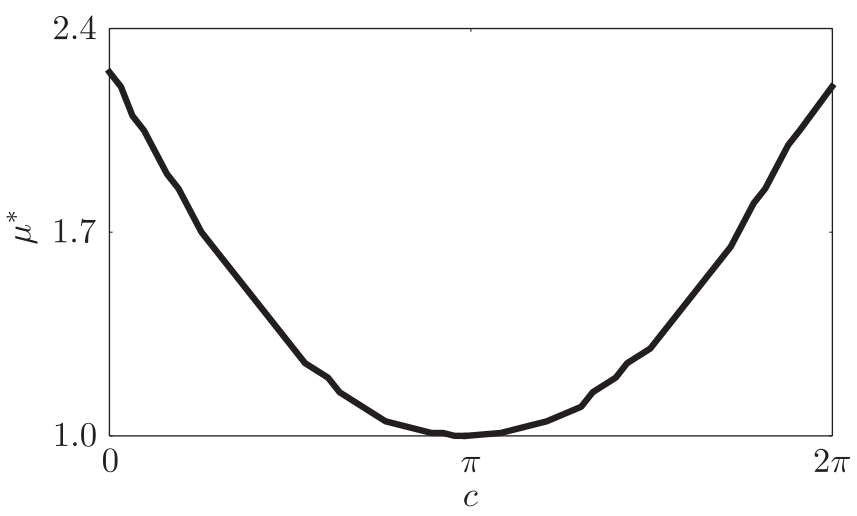

Fig. 2. $\mu^{*}(c)$. 
Substituting (2.6) in (2.5), we get the equation

$$
\varepsilon_{1} \dot{y}(t)+y(t)+\left(1+\varepsilon_{2}\right) y(t-1)+f\left(y(t-1) ; \varepsilon_{2}\right)=0,
$$

where $f\left(y(t-1) ; \varepsilon_{2}\right)=f\left(y ; \mu_{*}\left(c_{*}\right)+\mu\left(\varepsilon_{2}\right), c_{*}\right)=b_{2}\left(\varepsilon_{2}\right) y^{2}+b_{3}\left(\varepsilon_{2}\right) y^{3}+o\left(y^{3}\right)$, and

$$
b_{2}\left(\varepsilon_{2}\right)=x_{*}\left(\mu_{*}\left(c_{*}\right)+\mu\left(\varepsilon_{2}\right), c_{*}\right) / 2, b_{3}\left(\varepsilon_{2}\right)=-\left(1+\varepsilon_{2}\right) / 6 .
$$

The stability of the zero solution of Eq. (2.7) is determined by the behavior of the solutions of its linear part

$$
\varepsilon_{1} \dot{y}(t)+y(t)+\left(1+\varepsilon_{2}\right) y(t-1)=0,
$$

which is determined by the location of the roots of the characteristic equation

$$
P(\lambda ; \varepsilon) \equiv \varepsilon_{1} \lambda+1+\left(1+\varepsilon_{2}\right) \exp (-\lambda)=0\left(\varepsilon=\left(\varepsilon_{1}, \varepsilon_{2}\right)\right)
$$

of Eq. (2.9).

\section{The structure of the solutions of equation (2.9)}

Let us study the location of the roots of the characteristic equation (2.10) and the structure of the solutions of Eq. (2.9) for $|\varepsilon| \leqslant \varepsilon_{0}\left(|\varepsilon|=\left(\varepsilon_{1}^{2}+\varepsilon_{2}^{2}\right)^{1 / 2}\right)$. In this case the characteristic equation (2.10) has no roots lying on the real axis, therefore, we will take the area $\operatorname{Im} \lambda>0$.

Equation (2.10) in the form

$$
e^{\lambda}\left(\varepsilon_{1} \lambda+1\right)=-\left(1+\varepsilon_{2}\right)
$$

is equivalent to the sequence of equations

$$
e^{\lambda+\ln \left(1+\varepsilon_{1} \lambda\right)}=e^{\ln \left(1+\varepsilon_{2}\right)+i \pi k}, \quad k=1,3,5, \ldots,
$$

where $\ln z=\ln |z|+i \arg z(-\pi<\arg z<\pi), i=\sqrt{-1}$.

To determine the roots of Eq. (2.10), we consider the sequence of equations

$$
\lambda+\ln \left(1+\varepsilon_{1} \lambda\right)=\ln \left(1+\varepsilon_{2}\right)+i \pi k, \quad k=1,3,5, \ldots .
$$

Note that Eq. (2.10) can have only a finite number of roots of finite multiplicity in any bounded domain, since the left-hand side of Eq. (2.10) is an analytic function of the complex variable $\lambda$. In this case, the chain of roots of the form $\lambda_{p}(\varepsilon)=\gamma_{p}(\varepsilon)+i \sigma_{p}(\varepsilon)\left(\sigma_{p}(\varepsilon)>0\right),\left|\lambda_{p}(\varepsilon)\right| \rightarrow \infty$ $(p=1,2, \ldots)$ can exist in Eq. (2.10) only under the condition $\lim _{p \rightarrow \infty} \gamma_{p}(\varepsilon)=-\infty$, $\lim _{p \rightarrow \infty}\left|\sigma_{p}(\varepsilon) / \gamma_{p}(\varepsilon)\right|=\infty$ (see, for example, [9]). Therefore, there exists $\varphi_{0}>0$, which allows us to determine the region of existence of the roots of the equation as

$$
\Lambda_{\varphi_{0}}=\left\{\lambda: \varphi_{0}<\arg \lambda<\pi-\varphi_{0}\right\}
$$

Consider the equation

$$
F\left(\lambda ; \varepsilon_{1}\right) \equiv \lambda+\ln \left(1+\varepsilon_{1} \lambda\right)=w,
$$

where $\lambda \in \Lambda_{\varphi_{0}}, w \in \bar{W}_{x_{0}}=\left\{w:-x_{0} \leqslant \operatorname{Re} w \leqslant x_{0}, \operatorname{Im} w \geqslant \pi, x_{0}>0\right.$ being a small fixed number $\}$. Since $\left|\varepsilon_{1}\right| \leqslant \varepsilon_{0}<\sin \varphi_{0}$ and $\lambda \in \Lambda_{\varphi_{0}}\left|\varepsilon_{1}\right| /\left|1+\varepsilon_{1} \lambda\right| \leqslant q<1$, the inequality $m_{0}<\left|F_{\lambda}\left(\lambda ; \varepsilon_{1}\right)\right|<m_{\infty}$ holds uniformly with respect to $\varepsilon_{1}$ and $\lambda$, where $m_{0}, m_{\infty}$ are fixed 
positive constants. Thus, Eq. (3.2) for $\left|\varepsilon_{1}\right| \leqslant \varepsilon_{0}$ has a single solution $\lambda\left(w ; \varepsilon_{1}\right)$, which can be obtained through the iterative process

$$
\lambda_{p}+\ln \left(1+\varepsilon_{1} \lambda_{p-1}\right)=w, \quad p=1,2, \ldots, \quad \lambda_{0}=w .
$$

The iterative process converges uniformly with respect to $\varepsilon_{1}$ for $\left|\varepsilon_{1}\right| \leqslant \varepsilon_{0}$ and $w$ from any limited subdomain $\bar{W}_{x_{0}}$. The limit function

$$
\begin{aligned}
\lambda\left(w ; \varepsilon_{1}\right) & =w+\lambda^{1}\left(w ; \varepsilon_{1}\right), \\
\lambda^{1}\left(w ; \varepsilon_{1}\right) & =-\ln \left(1+\varepsilon_{1}\left(w-\ln \left(1+\varepsilon_{1}\left(w-\ln \left(1+\varepsilon_{1}(w-\ldots)\right)\right)\right)\right)\right.
\end{aligned}
$$

will be continuous in the aggregate of variables, analytic in $w \in W_{x_{0}}$ for each fixed $\varepsilon_{1}\left(\left|\varepsilon_{1}\right| \leqslant \varepsilon_{0}\right)$ and analytic in $\varepsilon_{1}$ for each fixed $w \in \bar{W}_{x_{0}}$.

Note that

$$
\lambda\left(w ; \varepsilon_{1}\right)-\lambda_{1}\left(w ; \varepsilon_{1}\right)=-\ln \left(1+\varepsilon_{1} w^{*}\right)+\ln \left(1+\varepsilon_{1} w\right)=\varepsilon_{1}^{2}\left(1+\varepsilon_{1} \lambda^{*}\right)^{-1} \ln \left(1+\varepsilon_{1} w^{*}\right),
$$

where according to $(3.3)-(3.4) \lambda_{1}\left(w ; \varepsilon_{1}\right)=w-\ln \left(1+\varepsilon_{1} w\right), w^{*}=w-\ln \left(1+\varepsilon_{1}(w-\ln (1+\right.$ $\left.\left.\left.+\varepsilon_{1}(w-\ldots)\right)\right)\right)$, the point $\lambda^{*}$ is on a straight line connecting points $w$ and $w^{*}$. Taking into account that the expression $\left|\left(1+\varepsilon_{1} \lambda^{*}\right)^{-1} \ln \left(1+\varepsilon_{1} w^{*}\right)\right|$ is limited uniformly with respect to $\varepsilon_{1}$ $\left(\left|\varepsilon_{1}\right| \leqslant \varepsilon_{0}\right)$ and $w \in \bar{W}_{x_{0}}$, we have the estimate

$$
\left|\lambda\left(w ; \varepsilon_{1}\right)-\lambda_{1}\left(w ; \varepsilon_{1}\right)\right|<K \varepsilon_{1}^{2} \quad(K>0) .
$$

This enables us to formulate the following assertion.

Theorem 1. There is a $\varepsilon_{0}>0$, that when $|\varepsilon|<\varepsilon_{0}$ the set of roots of Eq. (2.10) is defined by the formula

$$
\lambda_{k}(\varepsilon)=i \pi k+\ln \left(1+\varepsilon_{2}\right)+\lambda^{1}\left(i \pi k+\ln \left(1+\varepsilon_{2}\right) ; \varepsilon_{1}\right), \quad \lambda_{-k}(\varepsilon)=\bar{\lambda}_{k}(\varepsilon), \quad k=1,3,5, \ldots
$$

Hence, on the basis of (3.6) the following equality holds uniformly relative to $k$ :

$$
\begin{aligned}
& \lambda_{k}(\varepsilon)=\gamma_{k}(\varepsilon)+i\left(\pi k+\sigma_{k}(\varepsilon)\right), \\
& \gamma_{k}(\varepsilon)=\ln \left(1+\varepsilon_{2}\right)-\ln \left(\left(1+\varepsilon_{1} \ln \left(1+\varepsilon_{2}\right)\right)^{2}+\varepsilon_{1}^{2} \pi^{2} k^{2}\right) / 2+o(|\varepsilon|), \\
& \sigma_{k}(\varepsilon)=-\arccos \left(\left(1+\varepsilon_{1} \ln \left(1+\varepsilon_{2}\right)\right) /\left(\left(1+\varepsilon_{1} \ln \left(1+\varepsilon_{2}\right)\right)^{2}+\varepsilon_{1}^{2} \pi^{2} k^{2}\right)^{1 / 2}\right)+o(|\varepsilon|) .
\end{aligned}
$$

Thus, the problem of the stability of the solutions of Eq. (2.9) reduces to analyzing the functions $\gamma_{k}(\varepsilon)$, which are analytic at the point $\varepsilon=0$ and have the radius of convergence $r_{k}=O\left(k^{-1}\right)$. In this case we have

$$
\gamma_{k}(\varepsilon)=\varepsilon_{2}-\varepsilon_{1}^{2}(\pi k)^{2} / 2-\varepsilon_{1} \varepsilon_{2}-\varepsilon_{2}^{2} / 2+o\left(|\varepsilon|^{3}\right), \quad k=1,3,5, \ldots,
$$

i.e., for small $\varepsilon$ and $\varepsilon_{2}>\varepsilon_{1}^{2}(\pi k)^{2} / 2$ the $k$ th root of the characteristic equation (2.10) has a positive real part.

Let us now describe the structure of the solutions of Eq. (2.9). The phase space of Eq. (2.9) is the space of continuous real-valued functions $C[-1 ; 0]$, where the norm is defined in a standard way and denoted as $\|\cdot\|_{C}$. The solution of Eq. (2.9) (Eq. (2.5)) with $t \geqslant 0$ and initial condition $y_{0}(s) \in C[-1 ; 0]$ is the function $y(t+s ; \varepsilon)(-1 \leqslant s \leqslant 0)$, which turns Eq. (2.9) (Eq. (2.5)) into an identity equation and satisfies the initial condition $y\left(t_{0}+s ; \varepsilon\right) \equiv y_{0}(s)$. 
Introduce the Cauchy function $K(t ; \varepsilon)$ of Eq. $(2.9)$

$$
\begin{aligned}
& \varepsilon_{1} \dot{K}(t ; \varepsilon)+K(t ; \varepsilon)+\left(1+\varepsilon_{2}\right) K(t-1 ; \varepsilon)=0, \quad t>0 \\
& K(0 ; \varepsilon)=\varepsilon_{1}^{-1}, K(t ; \varepsilon) \equiv 0, \quad-1 \leqslant t<0 .
\end{aligned}
$$

Applying the Laplace transform to (3.10) and using the inversion formula, we have the expression

$$
\begin{aligned}
& K(t ; \varepsilon)=\frac{1}{2 \pi i} \int_{\bar{x}-i \infty}^{\bar{x}+i \infty} P^{-1}(\lambda ; \varepsilon) e^{\lambda t} d \lambda=\sum_{-\infty}^{\infty}\left(1+\varepsilon_{1}+\varepsilon_{1} \lambda_{k}(\varepsilon)\right)^{-1} e^{\lambda_{n}(\varepsilon) t}, \quad t>0, \\
& K(0 ; \varepsilon)=\varepsilon_{1}^{-1}, K(t ; \varepsilon) \equiv 0, \quad t<0 .
\end{aligned}
$$

In (3.11) $\bar{x}>0$ is a fixed number. The series (3.11) converges uniformly for every $0 \leqslant t \leqslant \bar{t}$.

Applying the Laplace transform to Eq. (2.9) and using the function (3.10) taking into account its representation in the form (3.11), the solution of Eq. (2.9) with initial condition $y_{0}(s)$ can be written as

$$
\begin{aligned}
y(t+s ; \varepsilon) & =T(t ; \varepsilon) y_{0}(s)= \\
& = \begin{cases}\varepsilon_{1} K(t+s) y_{0}(0)-\left(1+\varepsilon_{2}\right) \int_{-1}^{0} K\left(t+s-1-s_{1}\right) y_{0}\left(s_{1}\right) d s_{1}, & t+s \geqslant 0, \\
y_{0}(t+s), & t+s<0 .\end{cases}
\end{aligned}
$$

Formula (3.12) defines the family $T(t ; \varepsilon),(t \geqslant 0)$ of linear completely continuous operators acting in the space $C[-1,0]$ and forming a semigroup $T\left(t_{1}+t_{2} ; \varepsilon\right)=T\left(t_{1} ; \varepsilon\right) T\left(t_{2} ; \varepsilon\right)=$ $=T\left(t_{2} ; \varepsilon\right) T\left(t_{1} ; \varepsilon\right)(T(0)=I$ - unit operator $)$ of class $\mathbb{C}_{0}[10]$. According to (2.9) and (3.12), the generating (infinitesimal) semigroup operator $T(t ; \varepsilon)$ has the form

$$
A(\varepsilon) v= \begin{cases}d v / d s, & -1 \leqslant s<0 \\ -\varepsilon_{1}^{-1}\left(v(0)+\left(1+\varepsilon_{2}\right) v(-1)\right), & s=0\end{cases}
$$

with domain $D(A)=\left\{v(s) \in C^{1}[-1,0], \varepsilon_{1} v^{\prime}(0)+v(0)+\left(1+\varepsilon_{2}\right) v(-1)=0\right\}$.

$C^{k}[-1,0]$ is the space of $k$-times continuously differentiable real-valued functions, the norm in which is defined by the standard method. We denote by $\widetilde{C}[-1,0]$ the complex expansion of space $C[-1,0]$, where $\|v(s)\|_{\widetilde{C}}=\left\|\left(v_{1}(s)^{2}+v_{2}(s)^{2}\right)^{1 / 2}\right\|_{C}, v(s)=v_{1}(s)+i v_{2}(s)$.

The eigenvalues of the operator $A(\varepsilon)$ are $\lambda_{k}=\lambda_{k}(\varepsilon)$, and the corresponding eigenfunctions are $e_{k}(s ; \varepsilon)=e^{\lambda_{k}(\varepsilon) s} / P^{\prime}\left(\lambda_{k}(\varepsilon) ; \varepsilon\right)=e^{\lambda_{k}(\varepsilon) s} /\left(1+\varepsilon_{1}+\varepsilon_{1} \lambda_{k}(\varepsilon)\right), k= \pm 1, \pm 3, \ldots,\left\|e_{k}(s ; \varepsilon)\right\|_{\widetilde{C}} \sim 1$ for $k \rightarrow \infty$.

Along with (3.13) we introduce the operator

$$
A^{*}(\varepsilon) h= \begin{cases}d h / d s, & 0<s \leqslant 1, \\ \varepsilon_{1}^{-1}\left(h(0)+\left(1+\varepsilon_{2}\right) h(1)\right), & s=0\end{cases}
$$

in space $C[0,1]$ with domain $D\left(A^{*}\right)=\left\{h(s) \in C^{1}[0,1],-\varepsilon_{1} h^{\prime}(0)+h(0)+\left(1+\varepsilon_{2}\right) h(1)=0\right\}$. The operator (3.14) is conjugate to (3.13) with respect to the scalar product of S. N. Shimanov [11], 
which has the following form for Eq. (2.9):

$$
\langle v(s), h(s)\rangle=\varepsilon_{1} v(0) \bar{h}(0)-\left(1+\varepsilon_{2}\right) \int_{-1}^{0} \overline{h(\xi+1)} v(\xi) d \xi .
$$

The eigenvalues of the operator $A^{*}(\varepsilon)$ are $-\bar{\lambda}_{k}(\varepsilon)$, and the corresponding eigenfunctions are $h_{k}=$ $=h_{k}(s ; \varepsilon)=e^{-\bar{\lambda}_{k}(\varepsilon) s}$. The following orthogonality conditions are satisfied between the functions $e_{k}(s ; \varepsilon)$ and $h_{k}(s ; \varepsilon)$ :

$$
\left\langle e_{k_{1}}(s ; \varepsilon), h_{k_{2}}(s ; \varepsilon)\right\rangle=\delta_{k_{1} k_{2}},
$$

where $\delta_{k_{1} k_{2}}$ is the Kronecker symbol.

The expressions

$$
\begin{aligned}
& p_{k}(v ; \varepsilon)=\left\langle v(s), h_{k}(s, \varepsilon)\right\rangle=\int_{-1}^{0} v(s) d r_{k}(s, \varepsilon), \quad p_{-k}(v ; \varepsilon)=\bar{p}_{k}(v ; \varepsilon), \\
& r_{k}(s ; \varepsilon)= \begin{cases}\lambda_{k}^{-1}(\varepsilon), & s=0, \\
-\left(\varepsilon_{1}+\lambda_{k}^{-1}(\varepsilon)\right) e^{-\lambda_{k}(\varepsilon) s}, & -1 \leqslant s<0, \quad k=1,3, \ldots,\end{cases}
\end{aligned}
$$

define a sequence of linear continuous functionals in the space $\widetilde{C}[-1 ; 0]$, the norms of which are

$$
\left\|p_{k}(v ; \varepsilon)\right\|=\sup _{\|r(s)\|_{\widetilde{C}}=1}\left|p_{k}(v, \varepsilon)\right| \leqslant \stackrel{V}{V}_{-1}^{0}\left|r_{k}(s ; \varepsilon)\right|=O(1), \quad k \rightarrow \infty .
$$

Theorem 2. A system of functions $e_{k}(s, \varepsilon)$ is complete in $C[-1,0]$ in the following sense: there is no function $q(s) \in C[-1,0], q(s) \not \equiv 0$, for which $\left\langle q(s), h_{k}(s)\right\rangle=0, k= \pm 1, \pm 3, \ldots$

Proof. The operator $A^{-1}(\varepsilon)$ acting in $C[-1,0]$ has the following form:

$$
A^{-1}(\varepsilon) f(s)=\int_{-1}^{0} f\left(s_{1}\right) d_{s_{1}} R\left(s, s_{1} ; \varepsilon\right),
$$

where

$$
R\left(s, s_{1} ; \varepsilon\right)=\frac{1}{2+\varepsilon_{2}} \begin{cases}\left(1+\varepsilon_{2}\right)(s+1), & -1 \leqslant s_{1}<s \\ 1+\varepsilon_{2}-s_{1}, & s \leqslant s_{1}<0 \\ 1-\varepsilon_{1}+\varepsilon+2, & s_{1}=0\end{cases}
$$

is a completely continuous operator having single eigenvalues $\lambda_{k}^{-1}(\varepsilon), \quad k= \pm 1, \pm 3, \ldots$, $\left|\lambda_{1}^{-1}(\varepsilon)\right|>\left|\lambda_{2}^{-1}(\varepsilon)\right|>\ldots$, which correspond to the eigenfunctions $e_{k}(s ; \varepsilon), k= \pm 1, \pm 3, \ldots$. It follows from $A^{-1}(\varepsilon)$ that $A^{-1}(\varepsilon) f(s) \equiv 0$ if and only if $f(s) \equiv 0$. For the operator (3.18) in $C[-1,0]$ there is an equivalent norm \|\|$_{C}^{*}$, where $\left\|A^{-1}(\varepsilon)\right\|_{C}^{*}=\sup _{\|f\|_{C}^{*}=1}\left\|A^{-1}(\varepsilon) f(s)\right\|_{C}^{*}=$ $=\max _{k}\left|\lambda_{k}^{-1}(\varepsilon)\right|=\left|\lambda_{1}^{-1}(\varepsilon)\right|$ (see, for example, [12, pp. 15-16]).

Suppose we have found $q(s) \in C[-1,0], q(s) \not \equiv 0,\left\langle q(s), h_{k}(s)\right\rangle=0 k= \pm 1, \pm 3, \ldots$ We normalize $q(s) \rightarrow q(s) /\|q(s)\|_{C}^{*}$, i.e., $\|q(s)\|_{C}^{*}=1$. Denote $\left\|A^{-1}(\varepsilon) q(s)\right\|_{C}^{*}=\delta(\varepsilon)>0$. Consider all eigenvalues $\lambda_{k}^{-1}(\varepsilon)$ for which $\left|\lambda_{k}^{-1}(\varepsilon)\right| \geqslant \delta(\varepsilon) / 2,\left|\lambda_{k+1}^{-1}(\varepsilon)\right|<\delta(\varepsilon) / 2$, and there can be only a finite number of them $k= \pm 1, \pm 3, \ldots, \pm l$. Let $C_{l+1}[-1,0]$ denote the subspace of functions $C[-1,0]$ for which $\left\langle v(s), h_{k}(s, \varepsilon)\right\rangle=0, k= \pm 1, \pm 3, \ldots, \pm l$. In this case, $\sup _{\|f(s)\|_{C}^{*}=1, f(s) \in C_{l+1}[-1,0]}\left\|A^{-1}(\varepsilon) f(s)\right\|_{C}^{*}=\left|\lambda_{l+1}^{-1}(\varepsilon)\right|<\delta(\varepsilon) / 2$, but $q(s) \in C_{l+1}[-1,0]$ and $\left\|A^{-1}(\varepsilon) q(s)\right\|_{C}^{*}=\delta(\varepsilon)$. We have obtained the contradiction. The theorem is proved. 
Let $l_{2}$ denote the subspace of sequences of the form $z=\left(z_{1}, z_{-1}, z_{3}, z_{-3}, \ldots\right), z_{k} \in C, z_{-k}=\bar{z}_{k}$, $\|z\|_{l_{2}}^{2}=\sum_{k=1}^{\infty}\left|z_{k}\right|^{2}<\infty$. Let $l_{2}^{1}$ denote the subspace of $l_{2}$ sequences $z=\left(z_{1}, z_{-1}, z_{3}, z_{-3}, \ldots\right)$ for which $\|z\|_{l_{2}^{1}}^{2}=\sum_{k=1,3, \ldots}\left|\lambda_{k}(\varepsilon)\right|^{2}\left|z_{k}\right|^{2}<\infty$ and $\left\|\sum_{k= \pm 1, \pm 3, \ldots} z_{k} \lambda_{k}(\varepsilon) e_{k}(s ; \varepsilon)\right\|_{C}<\infty$. Denote by $D_{*}(A)$ the subspace $C[-1,0]$ of functions of the above-mentioned kind.

Theorem 3. Every solution $y(t+s ; \varepsilon)$ of Eq. (2.9), determined for $t>0$ with initial condition $y(s ; \varepsilon)=y_{0}(s) \in C[-h, 0]$, for $t \geqslant t_{0}\left(t_{0} \geqslant 3\right)$ can be represented in the form

$$
y(t+s ; \varepsilon)=\sum_{k= \pm 1, \pm 3, \ldots} z_{k}(t ; \varepsilon) e_{k}(s ; \varepsilon), \quad z(t ; \varepsilon)=\left(z_{1}(t ; \varepsilon), z_{-1}(t ; \varepsilon), \ldots\right) \in l_{2}^{1}
$$

where $z(t ; \varepsilon)$ is a solution on the equation

$$
\dot{z}=\Lambda(\varepsilon) z, \quad \Lambda(\varepsilon) z=\left(\lambda_{1}(\varepsilon) z_{1}, \lambda_{-1}(\varepsilon) z_{-1}, \ldots\right),
$$

in the space $l_{2}$ with initial condition $z\left(t_{0} ; \varepsilon\right)=\left(z_{1}\left(t_{0} ; \varepsilon\right), z_{-1}\left(t_{0} ; \varepsilon\right), \ldots\right)$, where

$$
z_{k}\left(t_{0} ; \varepsilon\right)=\left\langle y\left(t_{0}+s ; \varepsilon\right), h_{k}(s ; \varepsilon)\right\rangle, \quad k= \pm 1, \pm 3, \ldots
$$

Conversely, if $z(t ; \varepsilon)$ with $t \geqslant t_{0}$ is a solution of Eq. (3.20) with initial condition $z\left(t_{0} ; \varepsilon\right) \in l_{2}^{1}$, then the expression (3.19) determines for $t \geqslant t_{0}$ the solution of Eq. (2.9).

Proof. For $t_{0} \geqslant 3$ the solution of Eq. (2.9) is $y\left(t_{0}+s ; \varepsilon\right) \in C^{3}[-1,0]$ and $\varepsilon_{1} y^{\prime \prime \prime}\left(t_{0}\right)+y^{\prime \prime}\left(t_{0}\right)+$ $+\left(1+\varepsilon_{2}\right) y^{\prime \prime}\left(t_{0}-1\right)=0$. Hence, integrating by parts equation (3.21) taking into account (3.15) and (3.17), we have

$$
z_{k}\left(t_{0} ; \varepsilon\right)=\left\langle y^{\prime \prime \prime}\left(t_{0}+s ; \varepsilon\right), h_{k}(s ; \varepsilon)\right\rangle \lambda_{k}^{-3}(\varepsilon), \quad\left|z_{k}\left(t_{0} ; \varepsilon\right)\right|=O\left(k^{-3}\right), \quad k \rightarrow \infty .
$$

Thus, $z\left(t_{0} ; \varepsilon\right) \in l_{2}^{1}$ and $z_{k}(t, \varepsilon)=e^{\lambda_{k}(\varepsilon)\left(t-t_{0}\right)} z_{k}\left(t_{0} ; \varepsilon\right), k= \pm 1, \pm 3, \ldots$ Substituting $z_{k}(t ; \varepsilon)$ in (3.19), we note that the series and its derivative with respect to $t$ converge uniformly for $-1 \leqslant s \leqslant 0$ and satisfy Eq. (2.9) for $t \geqslant t_{0}$. Conversely, if $z(t ; \varepsilon)$ is the solution of (3.20) with initial condition $z\left(t_{0} ; \varepsilon\right) \in l_{2}^{1}$, then the function defined by the series $(3.19) y\left(t_{0}+s\right) \in D_{*}(A)$ and for $t>t_{0}$ satisfies Eq. (2.9), in which it is easily verified by direct substitution. The theorem is proved.

\section{The structure of solutions of Eq. (2.7) in a neighborhood of the zero equilibrium state}

Let us study the structure of solutions $y(t+s ; \varepsilon)$ of $(2.7)$, determined for $t>0$ with initial conditions $y(s ; \varepsilon)=y_{0}(s) \in S\left(R_{0}\right)=\left\{y(s) \in C[-1,0],\|y(s)\|_{C}<R_{0}\right\}$, i.e., belonging to a sphere of fixed radius $R_{0}$ with the center at the origin of the phase space. For $t>t_{0}\left(t_{0} \geqslant 3\right)$ $y(t+s ; \varepsilon) \in C^{3}[-1,0]$ and

$$
\begin{aligned}
\varepsilon_{1} \dddot{y}(t ; \varepsilon)+\ddot{y}(t ; \varepsilon)+\left(1+\varepsilon_{2}\right) \ddot{y}(t-1 ; \varepsilon)+f^{\prime \prime}(y(t-1 ; \varepsilon) & \left.; \varepsilon_{2}\right) \dot{y}^{2}(t-1 ; \varepsilon)+ \\
& +f^{\prime}\left(y(t-1 ; \varepsilon) ; \varepsilon_{2}\right) \ddot{y}(t-1 ; \varepsilon)=0 .
\end{aligned}
$$



have

Taking this into account and integrating by parts the scalar product (3.15) for $t \geqslant t_{0}$, we

$$
\begin{aligned}
& \left\langle y(t+s ; \varepsilon), h_{k}(s ; \varepsilon)\right\rangle=\lambda_{k}^{-1}(\varepsilon) f\left(y(t-1 ; \varepsilon) ; \varepsilon_{2}\right)+\lambda_{k}^{-2}(\varepsilon) f^{\prime}\left(y(t-1 ; \varepsilon) ; \varepsilon_{2}\right) \dot{y}(t-1 ; \varepsilon)+ \\
& +\lambda_{k}^{-3}(\varepsilon)\left(f^{\prime \prime}\left(y(t-1 ; \varepsilon) ; \varepsilon_{2}\right) \dot{y}^{2}(t-1 ; \varepsilon)+f^{\prime}\left(y(t-1 ; \varepsilon) ; \varepsilon_{2}\right) \ddot{y}(t-1 ; \varepsilon)+\right. \\
& \left.+\left\langle\dddot{y}(t+s ; \varepsilon), h_{k}(s ; \varepsilon)\right\rangle\right)=\lambda_{k}^{-1}(\varepsilon) f\left(y(t-1 ; \varepsilon) ; \varepsilon_{2}\right)+w_{k}(t ; \varepsilon), \quad k= \pm 1, \pm 3, \ldots
\end{aligned}
$$

Since

$$
\begin{aligned}
\lambda_{k}^{-1}(\varepsilon) e_{k}(s ; \varepsilon) & =\lambda_{k}^{-1}(\varepsilon)\left(1+\varepsilon_{1}+\varepsilon_{1} \lambda_{k}(\varepsilon)\right)^{-1} e^{\lambda_{k}(\varepsilon) s}= \\
& =\lambda_{k}(\varepsilon)^{-1}\left(1+\varepsilon_{1}+\varepsilon_{1} \lambda_{k}(\varepsilon)\right)^{-1}\left(1+\varepsilon \lambda_{k}(\varepsilon)\right)\left(1+\varepsilon_{2}\right)^{-1} e^{\lambda_{k}(\varepsilon)(s+1)}= \\
& =-\left(1+\varepsilon_{2}\right)^{-1}\left(\varepsilon_{1}+\lambda_{k}^{-1}(\varepsilon)\right)\left(1+\varepsilon_{1}+\varepsilon_{1} \lambda_{k}(\varepsilon)\right)^{-1} e^{\lambda_{k}(\varepsilon) s_{1}}, \quad 0 \leqslant s_{1} \leqslant 1,
\end{aligned}
$$

it follows from (3.11) and (3.17) that the series $\sum_{k= \pm 1, \pm 3, \ldots} \lambda_{k}^{-1}(\varepsilon) e_{k}(s ; \varepsilon)$ converges uniformly for $-1 \leqslant s \leqslant 0$ and $w(t ; \varepsilon)=\left(w_{1}(t ; \varepsilon), w_{-1}(t ; \varepsilon), \ldots,\right) \in l_{2}^{1}$, where $w_{k}(t ; \varepsilon)$ are determined in accordance with (4.2). This implies that, when $t \geqslant t_{0}$, the following representation holds for the solution $y(t+s ; \varepsilon)$ of Eq. (2.7):

$$
y(t+s ; \varepsilon)=\sum_{k= \pm 1, \pm 3, \ldots}\left(w_{k}(t ; \varepsilon)+\lambda_{k}^{-1}(\varepsilon) f\left(y(t-1 ; \varepsilon) ; \varepsilon_{2}\right) e_{k}(s ; \varepsilon)\right) .
$$

From (4.3) for $\left\|\sum_{k= \pm 1, \pm 3, \ldots} w_{k}(t ; \varepsilon) e_{k}(s ; \varepsilon)\right\|_{C} \leqslant r_{0}$, where $r_{0}$ is small, we get

$$
y(t-1 ; \varepsilon)=\sum_{k= \pm 1, \pm 3, \ldots} w_{k}(t ; \varepsilon) e_{k}(-1 ; \varepsilon)+g\left(\sum_{k= \pm 1, \pm 3, \ldots} w_{k}(t ; \varepsilon) e_{k}(-1 ; \varepsilon) ; \varepsilon\right) .
$$

Here $g(y ; \varepsilon)=g_{2}(\varepsilon) y^{2}+O\left(y^{2}\right)$ is an analytic function in a neighborhood of the point $y=0$.

Denote by $S\left(r_{0}\right)=\left\{z=\left(z_{1}, z_{-1}, \ldots\right) \in l_{2},\|z\|_{l_{2}}<r_{0}\right\}, S^{1}\left(r_{0}\right)=\left\{z=\left(z_{1}, z_{-1}, \ldots\right) \in l_{2}^{1}\right.$, $\left.\|z\|_{l_{2}^{1}}<r_{0}\right\}$. It follows from (4.4) that, when $w(t ; \varepsilon)=\left(w_{1}(t ; \varepsilon), w_{-1}(t ; \varepsilon), \ldots\right) \in S^{1}\left(r_{0}\right)$,

$$
\begin{aligned}
\left\langle y(t+s ; \varepsilon), h_{k}(s ; \varepsilon)\right\rangle & =w_{k}(t ; \varepsilon)+\lambda_{k}^{-1}(\varepsilon) f\left(\sum_{j= \pm 1, \pm 3, \ldots} w_{j}(t ; \varepsilon) e_{j}(-1 ; \varepsilon)+\right. \\
& \left.+g\left(\sum_{j= \pm 1, \pm 3, \ldots} w_{j}(t ; \varepsilon) e_{j}(-1 ; \varepsilon) ; \varepsilon\right) ; \varepsilon_{2}\right), \quad k= \pm 1, \pm 3 .
\end{aligned}
$$

Considering $f\left(y(t-1) ; \varepsilon_{2}\right)$ in $(2.5)$ as an inhomogeneous term of the linear equation (2.9), we write the solution $y(t+s ; \varepsilon)$ of Eq. $(2.5)$ for $t \geqslant t_{0}$ with initial condition $y_{0}(s ; \varepsilon)=y_{0}\left(t_{0}+s ; \varepsilon\right)$, according to (3.12) and using the function $K(t ; \varepsilon)$, in the following form:

$$
y(t+s ; \varepsilon)=\left\{\begin{array}{l}
\varepsilon_{1} K\left(t-t_{0}+s ; \varepsilon\right) y\left(t_{0} ; \varepsilon\right)-\left(1+\varepsilon_{2}\right) \int_{-1}^{0} K\left(t-t_{0}+s-1-s_{1}\right) y\left(t_{0}+s_{1} ; \varepsilon\right) d s_{1}+ \\
+\int_{t_{0}}^{t} K\left(t+s-t_{1} ; \varepsilon\right) f\left(y\left(t_{1}-1 ; \varepsilon\right) ; \varepsilon_{2}\right) d t_{1}, \quad t+s \geqslant t_{0}, \\
y_{0}(t+s ; \varepsilon), \quad t+s<t_{0} .
\end{array}\right.
$$


Hence, taking (3.10)-(3.11) and (4.4), (4.5) into account, we have the sequence of equalities

$$
\begin{aligned}
& w_{k}(t ; \varepsilon)+\lambda_{k}^{-1}(\varepsilon) f\left(\sum_{j= \pm 1, \pm 3, \ldots} w_{j}(t ; \varepsilon) e_{j}(-1 ; \varepsilon)+g\left(\sum_{j= \pm 1, \pm 3, \ldots} w_{j}(t ; \varepsilon) e_{j}(-1 ; \varepsilon) ; \varepsilon\right) ; \varepsilon_{2}\right)= \\
& \quad=\left\langle y\left(t_{0}+s ; \varepsilon\right), h_{k}(s ; \varepsilon)\right\rangle e^{\lambda_{k}(\varepsilon)\left(t-t_{0}\right)}-\int_{t_{0}}^{t} e^{\lambda_{k}(\varepsilon)\left(t-t_{1}\right)} f\left(\sum_{j= \pm 1, \pm 3, \ldots} w_{j}\left(t_{1} ; \varepsilon\right) e_{j}(-1, \varepsilon)+\right. \\
& \left.\quad+g\left(\sum_{j= \pm 1, \pm 3, \ldots} w_{j}\left(t_{1} ; \varepsilon\right) e_{j}(-1, \varepsilon) ; \varepsilon\right) ; \varepsilon_{2}\right) d t_{1}, \quad k= \pm 1, \pm 3, \ldots
\end{aligned}
$$

Differentiating Eqs. (4.7) for $t$,we obtain a system of differential equations in the space $l_{2}$ of the form

$$
\begin{aligned}
\dot{w}_{k} & +\lambda_{k}^{-1}(\varepsilon) f^{\prime}\left(\sum_{j= \pm 1, \pm 3, \ldots} w_{j} e_{j}\left(-1 ; \varepsilon_{2}\right)+g\left(\sum_{j= \pm 1, \pm 3, \ldots} w_{j} e_{j}(-1 ; \varepsilon) ; \varepsilon\right) ; \varepsilon_{2}\right) \times \\
& \times\left(1+g^{\prime}\left(\sum_{j= \pm 1, \pm 3, \ldots} w_{j} e_{j}(-1 ; \varepsilon) ; \varepsilon\right)\right) \sum_{j= \pm 1, \pm 3, \ldots} \dot{w}_{j} e_{j}(-1 ; \varepsilon)=\lambda_{k}(\varepsilon) w_{k} \\
& k= \pm 1, \pm 3, \ldots
\end{aligned}
$$

whose solution for $t \geqslant t_{0}$ is provided by the functions $w_{k}(t ; \varepsilon)(k= \pm 1, \pm 3, \ldots)$, defined in (4.2) with corresponding initial conditions $w_{k}\left(t_{0} ; \varepsilon\right)$. The opposite is also true. If $w(t ; \varepsilon)=$ $=\left(w_{1}(t ; \varepsilon), w_{-1}(t ; \varepsilon), \ldots\right) \in S^{1}\left(r_{0}\right)$ with $t \geqslant t_{0}$ is a solution of the system of differential equations (4.8), then according to (4.4), (4.5) for $t \geqslant t_{0}$ the series (4.3) converges uniformly in the area $\left\{-1 \leqslant s \leqslant 0, t_{0} \leqslant t \leqslant \bar{t}\right\}$ and, taking (4.2) into account, is a solution of (2.5).

Let us solve $(4.8)$ for $\dot{w}_{k}$. Multiply $(4.8)$ by $e_{k}(-1 ; \varepsilon)$ and sum by $k$. This allows us to obtain in explicit form an expression for $\sum_{k= \pm 1, \pm 3, \ldots} \dot{w}_{k} e_{k}(-1 ; \varepsilon)$. Note that $e_{k}(-1 ; \varepsilon)=e^{-\lambda_{k}(\varepsilon)}\left(1+\varepsilon_{1}+\right.$ $\left.+\varepsilon_{1} \lambda_{k}(\varepsilon)\right)^{-1}=\left(1+\varepsilon_{1} \lambda_{k}(\varepsilon)\right)\left(1+\varepsilon_{1}+\varepsilon_{1} \lambda_{k}(\varepsilon)\right)^{-1}\left(1+\varepsilon_{2}\right)^{-1} \sim 1$ for $k \rightarrow \infty$.

As a result, we obtain a system of differential equations equivalent to (4.8):

$$
\begin{aligned}
\dot{w}_{k} & =\lambda_{k}(\varepsilon) w_{k}-\lambda_{k}^{-1}(\varepsilon) f^{\prime}\left(\sum_{j= \pm 1, \pm 3, \ldots} w_{j} e_{j}(-1 ; \varepsilon)+g\left(\sum_{j= \pm 1, \pm 3, \ldots} w_{j} e_{j}(-1 ; \varepsilon) ; \varepsilon\right) ; \varepsilon_{2}\right) \times \\
& \times\left(1+g^{\prime}\left(\sum_{j= \pm 1, \pm 3, \ldots} w_{j} e_{j}(-1 ; \varepsilon) ; \varepsilon\right)\right) \sum_{p= \pm 1, \pm 3, \ldots} \lambda_{p}(\varepsilon) w_{p} e_{p}(-1 ; \varepsilon)\left(1+\lambda_{p}^{-1}(\varepsilon) \times\right. \\
& \times f^{\prime}\left(\sum_{j= \pm 1, \pm 3, \ldots} w_{j} e_{j}(-1 ; \varepsilon)+g\left(\sum_{j= \pm 1, \pm 3, \ldots} w_{j} e_{j}(-1 ; \varepsilon) ; \varepsilon\right) ; \varepsilon_{2}\right) \times \\
& \left.\times\left(1+g^{\prime}\left(\sum_{j= \pm 1, \pm 3, \ldots} w_{j} e_{j}(-1 ; \varepsilon) ; \varepsilon\right)\right)\right)^{-1}, \quad k= \pm 1, \pm 3, \ldots
\end{aligned}
$$

Note the following property of the function $\lambda_{k}(\varepsilon)$.

Theorem 4. There are $\varepsilon_{0}>0, c_{0}>0$ such that, when $0 \leqslant|\varepsilon| \leqslant \varepsilon_{0}$, the following inequalities hold true uniformly with respect to $k_{j}= \pm 1, \pm 3, \ldots(j=1, \ldots, 4)$ :

$$
\left|\lambda_{k_{1}}(\varepsilon)+\lambda_{k_{2}}(\varepsilon)+\lambda_{k_{3}}(\varepsilon)\right|, \quad\left|\lambda_{k_{1}}(\varepsilon)+\lambda_{k_{2}}(\varepsilon)+\lambda_{k_{3}}(\varepsilon)+\lambda_{k_{4}}(\varepsilon)\right| \geqslant c_{0},
$$

the second inequalities are true under the condition $k_{1}+k_{2}+k_{3}+k_{4} \neq 0$. 
Proof. The proof follows immediately from (3.7), (3.8). We first consider the first inequality (4.10). According to (3.8), $\left|\lambda_{k_{1}}(0)+\lambda_{k_{2}}(0)+\lambda_{k_{3}}(0)\right| \geqslant \pi$. Consider the case of equality, and let $k_{1}, k_{2}>0$, and $k_{3}=-k_{1}-k_{2}+1$. For $\varepsilon \neq 0$, according to (3.8), $\pi k_{j}-\pi / 2<\operatorname{Im} \lambda_{j}(\varepsilon)<\pi k_{j}$ and $\operatorname{Im} \lambda_{k_{j}} \rightarrow \pi k_{j}-\pi / 2$ for $k_{j} \rightarrow \infty(j=1,2)$. But then $\inf _{k_{1}, k_{2},|\varepsilon| \leqslant \varepsilon_{0}}\left(\operatorname{Im}\left(\lambda_{k_{1}}(\varepsilon)+\lambda_{k_{2}}(\varepsilon)+\right.\right.$ $\left.\left.+\lambda_{k_{3}}(\varepsilon)\right)\right)^{2}>0$. Thus, there exists $c_{0}>0$ with the properties specified in (4.10). Strict inequality and other combinations of $k_{1}, k_{2}, k_{3}$ are treated similarly. For the second inequality of (4.10) for $k_{1}+k_{2}+k_{3}+k_{3} \neq 0\left|\lambda_{k_{1}}(0)+\lambda_{k_{2}}(0)+\lambda_{k_{3}}(0)+\lambda_{k_{4}}(0)\right| \geqslant 2 \pi$. In accordance with (3.8) we have $\inf _{k_{1}, k_{2}, k_{3}, k_{4},|\varepsilon|<\varepsilon_{0}}\left(\operatorname{Im}\left(\lambda_{k_{1}}(\varepsilon)+\lambda_{k_{2}}(\varepsilon)+\lambda_{k_{3}}(\varepsilon)+\lambda_{k_{4}}(\varepsilon)\right)\right)^{2} \geqslant \pi^{2}$. The theorem is proved.

Taking into account inequalities (4.10), we can perform the normalizing change of variables in the system of equations (4.9) $w=z+c_{2}(z)+c_{3}(z): S^{1}\left(r_{0}\right) \rightarrow S^{1}\left(r_{0}\right)$ of the form

$$
\begin{aligned}
& w_{k}=z_{k}+\sum_{\left(k_{1}, k_{2}\right) \in \Omega_{2}} c_{k_{1} k_{2}}^{k}(\varepsilon) z_{k_{1}} z_{k_{2}}+\sum_{\left(k_{1}, k_{2}, k_{3}\right) \in \widetilde{\Omega}_{3}^{k}} c_{k_{1} k_{2} k_{3}}^{k}(\varepsilon) z_{k_{1}} z_{k_{2}} z_{k_{2}}, z_{-k}=\bar{z}_{k}, \\
& k= \pm 1, \pm 3, \ldots \text {, }
\end{aligned}
$$

where $\Omega_{2}=\left\{\left(k_{1}, k_{2}\right): k_{1}, k_{2}= \pm 1, \pm 3, \ldots, k_{1} \leqslant k_{2}\right\}, \widetilde{\Omega}_{3}^{k}=\left\{\left(k_{1}, k_{2}, k_{3}\right): k_{1}, k_{2}, k_{3}= \pm 1, \pm 3, \ldots\right.$, $\left.k_{1}+k_{2}+k_{3} \neq k, k_{1} \leqslant k_{2} \leqslant k_{3}\right\}$, leading it to a system of equations in the space $l_{2}$ of the form

$$
\dot{z}_{k}=\lambda_{k}(\varepsilon) z_{k}+\sum_{\left(k_{1}, k_{2}, k_{3}\right) \in \Omega_{3}^{k}} d_{k_{1} k_{2} k_{3}}(\varepsilon) z_{k_{1}} z_{k_{2}} z_{k_{3}}+Z_{k}^{*}(z ; \varepsilon) \equiv Z_{k}(z ; \varepsilon), \quad k= \pm 1, \pm 3, \ldots
$$

where $\Omega_{3}^{k}=\left\{\left(k_{1}, k_{2}, k_{3}\right): k_{1}, k_{2}, k_{3}= \pm 1, \pm 3, \ldots, k_{1}+k_{2}+k_{3}=k, k_{1} \leqslant k_{2} \leqslant k_{3}\right\}$, is the smooth operator with respect to the set of variables $Z(z ; \varepsilon)=\left(Z_{1}(z ; \varepsilon), Z_{-1}(z ; \varepsilon), \ldots\right)(Z(0 ; \varepsilon) \equiv 0)$ : $S^{1}\left(r_{0}\right) \rightarrow S\left(r_{0}\right)$ and $\left\|Z^{*}(z ; \varepsilon)\right\|_{l_{2}}=o\left(\|z\|_{l_{2}^{1}}^{3}\right)$.

To build a replacement (4.11) and Eqs. (4.12), substitute (4.11) in (4.9) with respect to (4.12) and equate coefficients of the same degree of $z_{k_{1}} z_{k_{2}}, z_{k_{1}} z_{k_{2}} z_{k_{3}}, k_{1}, k_{2}, k_{3}= \pm 1, \pm 3, \ldots$ in the resulting equations successively for each $k= \pm 1, \pm 3, \ldots$. As a result, we obtain a recursive sequence of algebraic equations, from which the coefficients $c_{k_{1} k_{2}}^{k}(\varepsilon), c_{k_{1} k_{2} k_{3}}^{k}(\varepsilon), d_{k_{1} k_{2} k_{3}}(\varepsilon)$ with the indicated properties are uniquely determined. From the conditions of final identities, for each $k$, the functions $Z_{k}^{*}(z ; \varepsilon)$ with the indicated properties are uniquely determined.

The system of equations (4.12) is called the normal form of Eq. (2.5) in the neighborhood of the zero equilibrium state normalized to cubic terms inclusive.

Note that the normalization of Eqs. (4.9) can be performed up to any odd power in $z_{k}$. In this case, for the functions $\lambda_{k}(\varepsilon)$, the relations of higher order similar to (4.10) are valid, and the normalized equations will not contain even powers of $z_{k}$.

Substituting the expressions (4.11) in (4.3) with (4.4), we get the final representation of the solutions of Eq. (2.5) with the initial conditions from the neighborhood of the zero equilibrium state.

Theorem 5. There are $R_{0}, r_{0}, \varepsilon_{0}>0$ such that, when $0<|\varepsilon|<\varepsilon_{0}$, every solution $y(t+s ; \varepsilon)$ of Eq. (2.5), determined for $t>0$ with initial condition $y_{0}(s ; \varepsilon) \in S\left(R_{0}\right)$, for $t \geqslant t_{0}\left(t_{0} \geqslant 3\right)$ and $t$ as long as $y(t+s ; \varepsilon) \in S\left(R_{0}\right)$, can be represented in the form

$$
y(t+s ; \varepsilon)=\sum_{k= \pm 1, \pm 3, \ldots}\left(z_{k}(t ; \varepsilon)+g_{k}(z(t ; \varepsilon) ; \varepsilon)\right) e_{k}(s ; \varepsilon)
$$


where the operator, which is smooth in the set of variables, has the form $g(z ; \varepsilon)=$ $=\left(g_{1}(z ; \varepsilon), g_{-1}(z ; \varepsilon), \ldots\right)(g(0 ; \varepsilon) \equiv 0): S^{1}\left(r_{0}\right) \rightarrow S^{1}\left(r_{0}\right)$ and $\|g(z ; \varepsilon)\|=o\left(\|z\|_{l_{2}^{1}}\right), z(t ; \varepsilon)=$ $=\left(z_{1}(t ; \varepsilon), z_{-1}(t ; \varepsilon), \ldots\right) \in S^{1}\left(r_{0}\right)$, and $z_{k}(t ; \varepsilon)(k= \pm 1, \pm 3, \ldots)$ are solutions of the system of equations (4.12) with initial conditions $z_{k}\left(t_{0} ; \varepsilon\right)$ defined by $\left\langle y\left(t_{0}+s ; \varepsilon\right), h_{k}(s ; \varepsilon)\right\rangle=z_{k}\left(t_{0} ; \varepsilon\right)+$ $+g_{k}\left(z\left(t_{0} ; \varepsilon\right) ; \varepsilon\right)$. Conversely, if $z(t ; \varepsilon) \in S^{1}\left(r_{0}\right)$ for $t \geqslant t_{0}$ are solutions of the system (4.12), then the function $y(t+s ; \varepsilon)$ defined according to (4.13) is a solution of Eq. (2.5).

Thus, the question of analyzing the bifurcations of periodic solutions of Eq. (2.5) from the zero equilibrium state with changing parameter $\varepsilon$ reduces to solving an analogous problem for the system of equations (4.12).

In what follows, the main part of the normal form (4.12) will be called the system of equations containing on the right-hand side terms that are not higher than those cubic in $z_{k}$.

The calculation of the main part of the normal form (4.12) can be performed directly from Eq. (2.5). Let us proceed from Eq. (2.5) to the equivalent initial boundary value problem

$$
\begin{gathered}
\frac{\partial u}{\partial t}=\frac{\partial u}{\partial s} \\
\left.\varepsilon_{1} \frac{\partial u}{\partial s}\right|_{s=0}=-u(0, t)-\left(1+\varepsilon_{2}\right) u(-1, t)-f\left(u(1, t) ; \varepsilon_{2}\right), \quad u(s, 0)=y_{0}(s)
\end{gathered}
$$

in the band $-1 \leqslant s \leqslant 0, t \geqslant 0$, putting $u(s, t)=y(t+s)$.

Introduce the function

$$
\begin{aligned}
u(s, z ; \varepsilon) & =\sum_{k= \pm 1, \pm 3, \ldots} z_{k} e_{k}(s ; \varepsilon)+\sum_{\left(k_{1}, k_{2}\right)=\Omega_{2}} z_{k_{1}} z_{k_{2}} u_{k_{1} k_{2}}(s ; \varepsilon)+ \\
& +\sum_{\left(k_{1}, k_{2}, k_{3}\right)=\Omega_{3}} z_{k_{1}} z_{k_{2}} z_{k_{3}} u_{k_{1} k_{2} k_{3}}(s ; \varepsilon)
\end{aligned}
$$

where $\Omega_{2}$ is defined in (4.11), $\Omega_{3}=\left\{\left(k_{1}, k_{2}, k_{3}\right): k_{1}, k_{2}, k_{3}= \pm 1, \pm 3, \ldots, k_{1} \leqslant k_{2} \leqslant k_{3}\right\}$, which operates from $s^{1}\left(r_{0}\right) \otimes\left\{|\varepsilon|<\varepsilon_{0}\right\}$ in $C[-1,0]$ and which depends smoothly on its variables, and the main part of the normal form (4.12) of Eq. (2.5)

$$
\dot{z}_{k}=\lambda_{k}(\varepsilon) z_{k}+\sum_{\left(k_{1} k_{2} k_{3}\right) \in \Omega_{3}^{k}} d_{k_{1} k_{2} k_{3}}(\varepsilon) z_{k_{1}} z_{k_{2}} z_{k_{3}} .
$$

Functions $u_{k_{1} k_{2}}(s ; \varepsilon), u_{k_{1} k_{2} k_{3}}(s ; \varepsilon), d_{k_{1} k_{2} k_{3}}(\varepsilon)$ should be determined. The condition for the trajectories of the system of equations (4.17) to belong to the boundary value problem (4.14)(4.15) by virtue of (4.16) takes the form

$$
\begin{aligned}
& \sum_{k= \pm 1, \pm 3, \ldots} \frac{\partial u(s, z ; \varepsilon)}{\partial z_{k}}\left(\lambda_{k}(\varepsilon) z_{k}+\sum_{\left(k_{1} k_{2} k_{3}\right) \in \Omega_{3}^{k}} d_{k_{1} k_{2} k_{3}}(\varepsilon) z_{k_{1}} z_{k_{2}} z_{k_{3}}\right)=\frac{\partial u(s, z ; \varepsilon)}{\partial s} \\
& \left.\varepsilon_{1} \frac{\partial u(s, z ; \varepsilon)}{\partial s}\right|_{s=0}=-u(0, z ; \varepsilon)-\left(1+\varepsilon_{2}\right) u(-1, z ; \varepsilon)-f\left(u(-1, z ; \varepsilon) ; \varepsilon_{2}\right) .
\end{aligned}
$$

Relations (4.18)-(4.19) determine the identities that should be satisfied uniformly over $|\varepsilon|<\varepsilon_{0}$ modulo $o\left(|z|_{l_{2}}^{3}\right)$. At the first degrees $z_{k}$ they are satisfied by the definition of functions $e_{k}(s ; \varepsilon)$ and $\lambda_{k}(\varepsilon)$. Relations (4.18)-(4.19) allow one to sequentially define the functions $u_{*}(s ; \varepsilon)$ and $d_{*}(\varepsilon)$ by equating the coefficients on the right and on the left for the same powers $z_{k}$. 
We equate the coefficients of (4.18)-(4.19) with $z_{k_{1}} z_{k_{2}}$. As a result, we obtain the boundary value problem

$$
\begin{gathered}
\left(\lambda_{k_{1}}(\varepsilon)+\lambda_{k_{2}}(\varepsilon)\right) u_{k_{1} k_{2}}(s ; \varepsilon)=\frac{d u_{k_{1} k_{2}}(s ; \varepsilon)}{d s} \\
\left.\varepsilon_{1} \frac{d u_{k_{1} k_{2}}(s ; \varepsilon)}{d s}\right|_{s=0}=-u_{k_{1} k_{2}}(0 ; \varepsilon)-\left(1+\varepsilon_{2}\right) u_{k_{1} k_{2}}(-1 ; \varepsilon)-p_{k_{1} k_{2}} b_{2}\left(\varepsilon_{2}\right) e_{k_{1}}(-1 ; \varepsilon) e_{k_{2}}(-1 ; \varepsilon)
\end{gathered}
$$

to determine $u_{k_{1} k_{2}}(s ; \varepsilon)$. In (4.21) $p_{k_{1} k_{2}}=1$ for $k_{1}=k_{2}$, and $p_{k_{1} k_{2}}=2$ for $k_{1} \neq k_{2}$. The solution (4.20)-(4.21) is defined uniquely

$$
u_{k_{1} k_{2}}(s ; \varepsilon)=-p_{k_{1} k_{2}} b_{2}\left(\varepsilon_{2}\right) e^{\left(\lambda_{k_{1}}(\varepsilon)+\lambda_{k_{2}}(\varepsilon)\right) s} / P\left(\lambda_{k_{1}}(\varepsilon)+\lambda_{k_{2}}(\varepsilon) ; \varepsilon\right) .
$$

Equating in (4.18)-(4.19) coefficients for $z_{k_{1}} z_{k_{2}} z_{k_{3}}$ yields the boundary value problem

$$
\begin{gathered}
e_{k}(s ; \varepsilon) d_{k_{1} k_{2} k_{3}}(\varepsilon)\left(\lambda_{k_{1}}(\varepsilon)+\lambda_{k_{2}}(\varepsilon)+\lambda_{k_{3}}(\varepsilon)\right) u_{k_{1} k_{2} k_{3}}(s ; \varepsilon)=\frac{d u_{k_{1} k_{2} k_{3}}(s ; \varepsilon)}{d s}, \quad k=k_{1}+k_{2}+k_{3}, \\
\left.\varepsilon_{1} \frac{d u_{k_{1} k_{2} k_{3}}(s ; \varepsilon)}{d s}\right|_{s=0}=-u_{k_{1} k_{2} k_{3}}(0 ; \varepsilon)-\left(1+\varepsilon_{2}\right) u_{k_{1} k_{2} k_{3}}(-1 ; \varepsilon)+f_{k_{1} k_{2} k_{3}}(\varepsilon),
\end{gathered}
$$

$$
\begin{aligned}
& f_{k_{1} k_{2} k_{3}}(\varepsilon)=-p_{k_{1} k_{2} k_{3}} b_{3}\left(\varepsilon_{2}\right) e_{k_{1}}(-1 ; \varepsilon) e_{k_{2}}(-1 ; \varepsilon) e_{k_{3}}(-1 ; \varepsilon)-2 b_{2}\left(\varepsilon_{2}\right) \times \\
& \quad \times\left\{\begin{array}{l}
e_{k_{1}}(-1 ; \varepsilon) u_{k_{2} k_{3}}(-1 ; \varepsilon), \text { if } k_{1}=k_{2}=k_{3}, \\
e_{k_{j}}(-1 ; \varepsilon) u_{k_{m} k_{p}}(-1 ; \varepsilon)+e_{k_{m}}(-1 ; \varepsilon) u_{k_{j} k_{p}}(-1 ; \varepsilon), \text { if } k_{j} \neq k_{m}=k_{p}, j, m, p=1,2,3, \\
e_{k_{1}}(-1 ; \varepsilon) u_{k_{2} k_{3}}(-1 ; \varepsilon)+e_{k_{2}}(-1 ; \varepsilon) u_{k_{1} k_{3}}(-1 ; \varepsilon)+e_{k_{3}}(-1 ; \varepsilon) u_{k_{1} k_{2}}(-1 ; \varepsilon), \text { if } k_{1} \neq k_{2} \neq k_{3},
\end{array}\right.
\end{aligned}
$$

$p_{k_{1} k_{2} k_{3}}=1$, if $k_{1}=k_{2}=k_{3}, p_{k_{1} k_{2} k_{3}}=3$, if $k_{1}=k_{2} \neq k_{3}$, or $k_{1}=k_{3} \neq k_{2}$, or $k_{2}=k_{3} \neq k_{1}$, $p_{k_{1} k_{2} k_{3}}=6$, if $k_{1} \neq k_{2} \neq k_{3}$.

The general solution of Eq. (4.22) has the form

$$
\begin{aligned}
u_{k_{1} k_{2} k_{3}}(s ; \varepsilon) & =e^{\lambda_{k_{1} k_{2} k_{3}}(\varepsilon) s}\left(c+d_{k_{1} k_{2} k_{3}}(\varepsilon)\left(1+\varepsilon_{1}+\varepsilon_{1} \lambda_{k}(\varepsilon)\right) \times\right. \\
& \left.\times\left(1-e^{\left(\lambda_{k}(\varepsilon)-\lambda_{k_{1} k_{2} k_{3}}(\varepsilon)\right) s}\right)\left(\lambda_{k}(\varepsilon)-\lambda_{k_{1} k_{2} k_{3}}(\varepsilon)\right)^{-1}\right),
\end{aligned}
$$

where we use the notation $\lambda_{k_{1} k_{2} k_{3}}(\varepsilon)=\lambda_{k_{1}}(\varepsilon)+\lambda_{k_{2}}(\varepsilon)+\lambda_{k_{3}}(\varepsilon)$ and $c$ is an arbitrary constant. Substituting (4.24) into the boundary condition (4.23), we get

$$
\begin{aligned}
d_{k_{1} k_{2} k_{3}}(\varepsilon) & =\left(\varepsilon_{1}+\left(1+\varepsilon_{2}\right)\left(e^{-\lambda_{k_{1} k_{2} k_{3}}(\varepsilon)}-e^{-\lambda_{k}(\varepsilon)}\right) /\left(\lambda_{k_{1} k_{2} k_{3}}(\varepsilon)-\lambda_{k}(\varepsilon)\right)\right)^{-1} \times \\
& \times\left(1+\varepsilon_{1}+\lambda_{k}(\varepsilon)\right) f_{k_{1} k_{2} k_{3}}(\varepsilon) .
\end{aligned}
$$

The condition for the function $u_{k_{1} k_{2} k_{3}}(s ; \varepsilon)$ to be continuous in $\varepsilon$ with $0 \leqslant|\varepsilon| \leqslant\left|\varepsilon_{0}\right|$ gives $c=0$, which uniquely defines it by equality (4.24).

When constructing the normal form (4.12) up to order $2 m+1$, where $m>1$, of the corresponding order, one takes expansions (4.16) and (4.17). The algorithmic part repeats the foregoing. 


\section{Analysis of the normal form of Eq. (2.7)}

We transform the system of equations (4.12) to polar coordinates by setting $z_{k}=\rho_{k} e^{i \tau_{k}}$ $\left(\rho_{k} \geqslant 0,0 \leqslant \tau_{k}<2 \pi\right)$. As a result, we obtain the following system of equations:

$$
\begin{gathered}
\dot{\rho}_{k}=\gamma_{k}(\varepsilon) \rho_{k}+R_{k}^{(3)}(\rho, \tau ; \varepsilon)+R_{k}^{(*)}(\rho, \tau ; \varepsilon), \\
\dot{\tau}_{k}=\pi k+\sigma_{k}(\varepsilon)+\left(T_{k}^{(3)}(\rho, \tau ; \varepsilon)+T_{k}^{(*)}(\rho, \tau ; \varepsilon)\right) / \rho_{k}, \quad k=1,3, \ldots,
\end{gathered}
$$

where $\gamma_{k}(\varepsilon)$ and $\sigma_{k}(\varepsilon)$ are defined in $(3.8), \rho=\left(\rho_{1}, \rho_{3}, \ldots\right),\|\rho\|_{l_{2}^{1}}^{2}=\sum_{k=1,3, \ldots} k^{2} \rho_{k}^{2}<\infty, \tau=$ $=\left(\tau_{1}, \tau_{3}, \ldots\right)$, the functionals $R_{k}^{(3)}(\cdot), R_{k}^{(*)}(\cdot), T_{k}^{(3)}(\cdot), T_{k}^{(*)}(\cdot)$ smoothly depend on incoming variables and parameters and are $2 \pi$-periodic in $\tau_{j}$, with $R_{k}^{(3)}(\cdot)$ and $T_{k}^{(3)}(\cdot)$ being cubic forms in $\rho$, and $\left|R_{k}^{*}(\cdot)\right|,\left|T_{k}^{*}(\cdot)\right|=o\left(\|\rho\|_{l_{2}^{1}}^{3}\right)$.

The structure of the system (5.1)-(5.2) allows one to introduce one "fast" variable and a countable number of "slow" variables. How this can be done will be shown first by the example of a "truncate" system. Consider the main part of the normal form (4.17), setting $z_{k}=0, k= \pm 5, \pm 7, \ldots$ As a result, we have the system of equations

$$
\begin{aligned}
& \dot{z}_{1}=\lambda_{1}(\varepsilon) z_{1}+d_{-111}(\varepsilon) z_{-1} z_{1}^{2}+d_{-313}(\varepsilon) z_{-3} z_{1} z_{3}+d_{-1-13}(\varepsilon) z_{-1}^{2} z_{3}, \\
& \dot{z}_{3}=\lambda_{3}(\varepsilon) z_{3}+d_{-113}(\varepsilon) z_{-1} z_{1} z_{3}+d_{-333}(\varepsilon) z_{-3} z_{3}^{2}+d_{111}(\varepsilon) z_{1}^{3} .
\end{aligned}
$$

Equations for $z_{-1}, z_{-3}$ are obtained by conjugation of (5.3)-(5.4) taking into account the equalities $\bar{\lambda}_{k}(\varepsilon)=\lambda_{-k}(\varepsilon), \bar{z}_{k}(\varepsilon)=z_{-k}(\varepsilon)$. Denoting $d_{*}(\varepsilon)=a_{*}(\varepsilon)+i b_{*}(\varepsilon), A_{*}(\varepsilon)=\left|d_{*}(\varepsilon)\right|, \beta_{*}(\varepsilon)=$ $=\arg d_{*}(\varepsilon) \in[0,2 \pi)$, we change in (5.3)-(5.4) to polar variables $\rho_{1}, \rho_{3}, \tau_{1}, \tau_{3}$. This yields

$$
\begin{aligned}
& \dot{\rho}_{1}=\left(\gamma_{1}(\varepsilon)+a_{-111}(\varepsilon) \rho_{1}^{2}+a_{-313}(\varepsilon) \rho_{3}^{2}\right) \rho_{1}+A_{-1-13}(\varepsilon) \cos \left(-3 \tau_{1}+\tau_{3}+\beta_{-1-13}(\varepsilon)\right) \rho_{1}^{2} \rho_{3}, \\
& \dot{\rho}_{3}=\left(\gamma_{3}(\varepsilon)+a_{-1-13}(\varepsilon) \rho_{1}^{2}+a_{-33-3}(\varepsilon) \rho_{3}^{2}\right) \rho_{3}+A_{111}(\varepsilon) \cos \left(3 \tau_{1}-\tau_{3}+\beta_{111}(\varepsilon)\right) \rho_{1}^{3}, \\
& \dot{\tau}_{1}=\pi+\sigma_{1}(\varepsilon)+b_{-111}(\varepsilon) \rho_{1}^{2}+b_{-313}(\varepsilon) \rho_{3}^{2}+A_{-1-13}(\varepsilon) \sin \left(-3 \tau_{1}+\tau_{3}+\beta_{-1-13}(\varepsilon)\right) \rho_{1} \rho_{3}, \\
& \dot{\tau}_{3}=3 \pi+\sigma_{3}(\varepsilon)+b_{-1-13}(\varepsilon) \rho_{1}^{2}+b_{-333}(\varepsilon) \rho_{3}^{2}+A_{111}(\varepsilon) \sin \left(3 \tau_{1}-\tau_{3}+\beta_{111}(\varepsilon)\right) \rho_{1}^{3} / \rho_{3} .
\end{aligned}
$$

Let us pass in (5.5)-(5.8) to variables $\rho_{1}, \rho_{3}, \theta_{1}=-3 \tau_{1}+\tau_{3}$ and $\tau=\tau_{1}$. As a result, we obtain the system of equations

$$
\begin{aligned}
\dot{\rho}_{1} & =\left(\gamma_{1}(\varepsilon)+a_{-111}(\varepsilon) \rho_{1}^{2}+a_{-313}(\varepsilon) \rho_{3}^{2}\right) \rho_{1}+A_{-1-13}(\varepsilon) \rho_{1}^{2} \cos \left(\theta_{1}+\beta_{-1-13}(\varepsilon)\right) \rho_{1}^{2} \rho_{3}, \\
\dot{\rho}_{3} & =\left(\gamma_{3}(\varepsilon)+a_{-1-13}(\varepsilon) \rho_{1}^{2}+a_{-33-3}(\varepsilon) \rho_{3}^{2}\right) \rho_{3}+A_{111}(\varepsilon) \rho_{1}^{2} \cos \left(-\theta_{1}+\beta_{111}(\varepsilon)\right) \rho_{1}^{3}, \\
\dot{\theta}_{1} & =\delta_{1}(\varepsilon)+\left(-3 b_{-111}(\varepsilon)+b_{-1-13}(\varepsilon)\right) \rho_{1}^{2}+\left(-3 b_{-313}(\varepsilon)+b_{-333}(\varepsilon)\right) \rho_{3}^{2}- \\
& -3 A_{-1-13}(\varepsilon) \sin \left(\theta_{1}+\beta_{-1-13}(\varepsilon)\right) \rho_{1} \rho_{3}+A_{111}(\varepsilon) \sin \left(-\theta_{1}+\beta_{111}(\varepsilon)\right) \rho_{1}^{3} / \rho_{3},
\end{aligned}
$$

for "slow" variables, where $\delta_{1}(\varepsilon)=-3 \sigma_{1}(\varepsilon)+\sigma_{3}(\varepsilon)$, and an equation for the "fast" variable

$$
\dot{\tau}=\pi+\sigma_{1}(\varepsilon)+b_{-111}(\varepsilon) \rho_{1}^{2}+b_{-313}(\varepsilon) \rho_{3}^{2}+A_{-1-13}(\varepsilon) \sin \left(\theta_{1}+\beta_{-1-13}(\varepsilon)\right) \rho_{1} \rho_{3} .
$$

We note that the right-hand side of (5.9)-(5.11) does not depend on $\tau$.

Suppose now that in (4.17) all $z_{k}(t) \neq 0$. We can introduce the variables $\theta_{k}$ in more than one way, but they are all related to each other by linear relations. One of the ways to introduce $\theta_{k}$ is as follows. As a "fast" variable $\tau$ we take $\tau_{1}$ and consider the "truncated" finite-dimensional 
systems, consistently assuming in (4.17) $z_{k}=0, k= \pm 5, \pm 7, \ldots$, then $k= \pm 7, \pm 9, \ldots$, etc. The first case has been considered above. In the second case, two new equations are added to the system (5.3)-(5.4) for variables $z_{5}$ and $z_{-5}$. In this case, a resonant monomial appears on the right-hand side of the equation for $z_{1} z_{5} z_{-3} z_{-1}$ (in the equation for $z_{-1}$ the resonant monomial $z_{-5} z_{3} z_{1}$ appears). When moving to polar coordinates, this leads to the appearance of terms that depend on the expression $\tau_{5}-\tau_{3}-2 \tau_{1}=\theta_{3}$, which we take as a new "slow" variable. As a result, we have two additional "slow" variables $\rho_{5}, \theta_{3}$. The system of equations for $\rho_{1}, \rho_{3}, \rho_{5}, \theta_{1}, \theta_{3}$ will have a form similar to (5.9)-(5.11), the right-hand side of which will not depend on $\tau$ either. On the right-hand side of Eq. (5.12), new terms appear for $\tau$. When considering the next "truncated" system on the right-hand side of the equation for $z_{1}$, we obtain a resonant monomial $z_{7} z_{-5} z_{-1}$, and changing to polar coordinates, we obtain a term depending on the expression $\tau_{7}-\tau_{5}-2 \tau_{1}=\theta_{5}$. We have two new "slow" variables $\rho_{7}, \theta_{5}$. In the general case, in the next step, adding equations for $z_{k_{0}}$ and $z_{-k_{0}}$, we obtain in the equations for $z_{1}$ and $z_{-1}$ new monomials $z_{k_{0}} z_{-k_{0}+2} z_{-1}$ and $z_{-k_{0}} z_{k_{0}-2} z_{1}$, respectively, and moving to the polar coordinates, we obtain terms depending on $\tau_{k_{0}}-\tau_{k_{0}-2}-2 \tau_{1}=\theta_{k_{0}-2}$. We have two new "slow" variables $\rho_{k_{0}}, \theta_{k_{0}-2}$. Continuing this process, we change in (4.17) to the "slow" variables $\rho=$ $=\left(\rho_{1}, \rho_{3}, \ldots\right), \theta=\left(\theta_{1}, \theta_{3}, \ldots\right)$ and the "fast" variable $\tau$. The corresponding transformation to "slow" and "fast" variables in (5.1), (5.2) gives the following system of differential equations for their determination

$$
\begin{aligned}
\dot{\rho}_{k} & =\gamma_{k}(\varepsilon) \rho_{k}+R_{k}(\rho, \theta ; \varepsilon)+R_{k}^{*}(\rho, \theta, \tau ; \varepsilon), \\
\dot{\theta}_{k} & =\delta_{k}(\varepsilon)+\Theta_{k}(\rho, \theta ; \varepsilon)+\Theta_{k}^{*}(\rho, \theta, \tau ; \varepsilon), \quad k=1,3, \ldots, \\
\dot{\tau} & =\pi+\sigma_{1}(\varepsilon)+T(\rho, \theta ; \varepsilon)+T^{*}(\rho, \theta, \tau ; \varepsilon),
\end{aligned}
$$

where the functionals $R_{k}(\cdot), R_{k}^{*}(\cdot), \Theta_{k}(\cdot), \Theta_{k}^{*}(\cdot), T(\cdot), T^{*}(\cdot)-2 \pi$ are periodic in $\theta_{k}$, the other properties and properties of the function $\delta_{k}(\varepsilon)\left(\delta_{k}(0)=0\right)$ are determined by the properties of the functions and functionals appearing in (5.1)-(5.2).

The phase space of the system of equations (5.13)-(5.15) is the product of spaces $l_{2} \otimes c \times R$, here $l_{2}=\left\{\rho=\left(\rho_{1}, \rho_{3}, \ldots\right), \rho_{k} \geqslant 0,\|\rho\|_{l_{2}}^{2}=\sum_{k=1,3, \ldots} \rho_{k}^{2}<\infty\right\}, c=\left\{\theta=\left(\theta_{1}, \theta_{3}, \ldots\right),\|\theta\|_{c}=\right.$ $\left.=\sup _{k}\left|\theta_{k}\right|<\infty\right\}$. The domain of the right-hand side of (5.13)-(5.15) is a product of spaces $l_{2}^{1} \otimes c_{0} \times R$, where $l_{2}^{1}=\left\{\rho \in l_{2}, \rho_{k}>0,\|\rho\|_{l_{2}^{1}}^{2}=\sum_{k=1,3, \ldots} k^{2} \rho_{k}^{2}<\infty\right\}, c_{0}=\left\{\theta=\left(\theta_{1}, \theta_{3}, \ldots\right) \in c\right.$, $\left.0 \leqslant \theta_{k}<2 \pi\right\}$. setting

We introduce variables $\zeta \geqslant 0$ and $\pi / 2<\psi<\pi / 2$ in the domain $\left\{\left(\varepsilon_{1}, \varepsilon_{2}\right), \varepsilon_{1}>0,|\varepsilon|<\varepsilon_{0}\right\}$,

$$
\zeta=\left(\varepsilon_{1}^{2}+\left|\varepsilon_{2}\right|\right)^{1 / 2}, \quad \varepsilon_{1}=\zeta \cos \psi, \quad \varepsilon_{2}=\zeta^{2} \sin ^{2} \psi \operatorname{sign} \psi .
$$

Substituting (5.16) into (5.13)-(5.15) and normalizing $\rho_{k} \rightarrow \zeta \rho_{k}$, we get the system of equations

$$
\begin{aligned}
\dot{\rho}_{k} & =\gamma_{k}(\psi, \zeta) \rho_{k}+\zeta^{2} R_{k}(\rho, \theta ; \psi, \zeta)+\zeta^{3} R_{k}^{*}(\rho, \theta, \tau ; \psi, \zeta), \\
\dot{\theta}_{k} & =\delta_{k}(\psi, \zeta)+\zeta^{2} \Theta_{k}(\rho, \theta ; \psi, \zeta)+\zeta^{3} \Theta_{k}^{*}(\rho, \theta, \tau ; \psi, \zeta), \quad k=1,3, \ldots, \\
\dot{\tau} & =\pi+\sigma_{1}(\psi, \zeta)+\zeta^{2} T(\rho, \theta ; \psi, \zeta)+\zeta^{3} T^{*}(\rho, \theta, \tau ; \psi, \zeta),
\end{aligned}
$$

in which the properties of functions and functionals are determined by the properties of the functions and functionals of (5.13)-(5.15).

Since, according to (3.8) and (5.16), the equation $\gamma_{k}(\psi, \zeta)=O\left(\zeta^{2}\right), \delta_{k}(\psi, \zeta)=O\left(\zeta^{3}\right)$ is satisfied uniformly with respect to $k$, Eqs. (5.17) and (5.18) justify the name of equations of "slow" variables, and (5.19), the name of the equation of a "fast" variable. 
Consider the main part of the system of equations of "slow" variables (5.17)-(5.18). Rescaling time by $t \rightarrow t / \zeta^{2}$, we get

$$
\begin{aligned}
\dot{\rho}_{k} & =\gamma_{k}^{*}(\psi, \zeta) \rho_{k}+R_{k}(\rho, \theta ; \psi, \zeta), \\
\dot{\theta}_{k} & =\delta_{k}^{*}\left((\psi, \zeta)+\Theta_{k}(\rho, \theta ; \psi, \zeta), \quad k=1,3, \ldots,\right.
\end{aligned}
$$

where, according to $(3.8)$ and $(5.16), \gamma_{k}^{*}(\psi, \zeta)=\gamma_{k}(\psi, \zeta) / \zeta^{2}, \delta_{k}^{*}(\psi, \zeta)=\delta_{k}(\psi, \zeta) / \zeta^{2}$ are continuous functions $\pi / 2 \leqslant \psi \leqslant-\pi / 2,0 \leqslant \zeta \leqslant \zeta_{0}$. In this case, $\gamma_{k}^{*}(\psi, 0)=\gamma_{k}(\psi)=\sin ^{2} \psi \operatorname{sign} \psi-$ $-\pi^{2} k^{2} \cos ^{2} \psi / 2, \delta_{k}^{*}(\psi, 0)=0$.

Consider the system of nonlinear equations in $l_{2} \otimes c$

$$
\begin{gathered}
\gamma_{k}(\psi) \rho_{k}+R_{k}(\rho, \theta)=0 \quad\left(R_{k}(\rho, \theta) \equiv R_{k}(\rho, \theta ; \psi, 0)=0\right), \\
\Theta_{k}(\rho, \theta)=0 \quad\left(\Theta_{k}(\rho, \theta) \equiv \Theta_{k}(\rho, \theta ; \psi, 0)=0\right), \quad k=1,3, \ldots,
\end{gathered}
$$

where $(\rho, \theta) \in E_{0}^{1}=l_{2}^{1} \otimes c_{0}$. In (5.22) the operator $\gamma(\psi) \rho=\left(\gamma_{1}(\psi) \rho_{1}, \gamma_{3}(\psi) \rho_{3}, \ldots\right)$ is considered on the basis of $(5.20)$ as symmetrically extended to $l_{2}^{1}$.

Let $\left(\rho^{*}(\psi), \theta^{*}(\psi)\right) \in l_{2}^{1} \otimes c_{0}$ be the solution of the system (5.22)-(5.23).

We introduce the infinite matrix

$$
B(\psi)=\left(\begin{array}{cc}
\gamma_{k}(\psi) \delta_{k j}+\partial R_{k} / \partial \rho_{j} & \partial R_{k} / \partial \theta_{j} \\
\partial \Theta_{k} / \partial \rho_{j} & \partial \Theta_{k} / \partial \theta_{j}
\end{array}\right) \quad(k, j=1,3, \ldots),
$$

calculated at the point $\rho^{*}(\psi), \theta^{*}(\psi)$, where $\delta_{k_{j}}$ is the Kronecker symbol. The matrix (5.24) defines the linear operator

$$
B(\psi) v(v=(\rho, \theta)): E^{1}=l_{2}^{1} \otimes c_{0} \rightarrow E=l_{2} \otimes c,
$$

$\|v\|_{E}=\|\rho\|_{l_{2}}+\|\theta\|_{c},\|v\|_{E^{1}}=\|\rho\|_{l_{2}^{1}}+\|\theta\|_{c}$.

Let $\mu_{k}(\psi)\left(B(\psi) v_{k}(\psi)=\mu_{k}(\psi) v(\psi)\right)$ be the eigenvalue of the operator (5.25). We show that the operator (5.25) has a countable number of eigenvalues $\mu_{k}(\psi)$ which can be numbered in increasing order of their moduli and that any bounded domain of the complex plane contains only a finite number of eigenvalues of finite multiplicity. The limit can only be an infinity point, with $\lim _{k \rightarrow \infty} \operatorname{Re} \mu_{k}(\psi)=-\infty$.

Indeed, the functions

$$
\begin{aligned}
z_{1}(t ; \psi, \zeta) & =\zeta z_{1}^{*}(\tau ; \psi)=\zeta \rho_{1}^{*}(\psi) e^{i \tau}, \quad z_{3}(t ; \psi, \zeta)=\zeta z_{3}^{*}(\tau ; \psi)=\zeta \rho_{3}^{*}(\psi) e^{i\left(3 \tau+\theta_{1}^{*}(\psi)\right)} \\
z_{5}(t ; \psi, \zeta) & =\zeta z_{5}^{*}(\tau ; \psi)=\zeta \rho_{5}^{*}(\psi) e^{i\left(5 \tau+\theta_{1}^{*}(\psi)+\theta_{3}^{*}(\psi)\right)}, \ldots, z_{-k}(t ; \psi, \zeta)=\bar{z}_{k}(t ; \psi, \zeta) \\
k & =1,3, \ldots,
\end{aligned}
$$

determine the periodic solution of the following system of differential equations:

$$
\dot{z}_{k}=\left(i \pi k(1-\zeta \cos \psi)+\zeta^{2} \gamma_{k}(\psi)\right) z_{k}+\sum_{\left(k_{1}, k_{2}, k_{3}\right) \in \Omega_{3}^{k}} d_{k_{1} k_{2} k_{3}}(0) z_{k_{1}} z_{k_{2}} z_{k_{3}}, \quad k= \pm 1, \pm 3, \ldots
$$

$\left(\Omega_{3}^{k}\right.$ and $d_{k_{1} k_{2} k_{3}}(0)$ are determined, respectively, in (4.12) and (4.25)), which can be easily checked by direct substitution of (5.27) into (5.28), taking into account the substitutions for $\rho^{*}(\psi), \theta^{*}(\psi)$ 
and Eqs. (5.22), (5.23) for $\rho^{*}(\psi), \theta^{*}(\psi)$. The system of equations (5.28) is abstractly parabolic in the complex space $l_{2}$ with the domain $l_{2}^{1}$. For such systems of equations the monodromy operator constructed for the periodic solution (5.27) is completely continuous, containing a countable number of eigenvalues (multipliers) $\rho_{k}(\psi), \lim _{k \rightarrow \infty}\left|\rho_{k}(\psi)\right|=0$. The operator (5.25) determines the characteristic exponents of the periodic solution (5.27). This leads to all the listed properties of $\mu_{k}(\psi)$. Substituting $\rho^{*}(\psi), \theta^{*}(\psi)$ into (5.19), we obtain by virtue of (3.8) and (4.25)

$$
\dot{\tau}=\pi-\zeta \cos \psi+\zeta^{2} \cos ^{2} \psi+O\left(\zeta^{3}\right) .
$$

Theorem 6. Suppose that for some $\psi$ the system of equations (5.22)-(5.23) has a solution $\left(\rho^{*}(\psi), \theta^{*}(\psi)\right) \in l_{2}^{1} \otimes c_{0}$ and the matrix (5.24) defines the operator (5.25), which has no eigenvalues lying on the imaginary axis of the complex plane. Then there exists a $\zeta_{0}>0$ such that for $0<\zeta \leqslant \zeta_{0} E q$. (2.5), where $\varepsilon_{1}$ and $\varepsilon_{2}$ are defined according to (5.16), has a periodic solution $y^{*}(t+s ; \varepsilon)=y^{*}(t+s ; \psi, \zeta)$ of the form

$$
y^{*}(t ; \psi, \zeta)=\zeta \sum_{k= \pm 1, \pm 3} z_{k}^{*}(\tau ; \psi)-\zeta^{2} b_{2}(0) / 2 \sum_{\left(k_{1}, k_{2}\right) \in \Omega_{2}} p_{k_{1} k_{2}} z_{k_{1}}^{*}(\tau ; \psi) z_{k_{2}}^{*}(\tau ; \psi)+O\left(\zeta^{3}\right)
$$

where $b_{2}\left(\varepsilon_{2}\right), \Omega_{2}, p_{k_{1} k_{2}}, z_{k}^{*}(\tau ; \psi)$ and $\tau$ are, respectively, defined in (2.8), (4.11), (4.20), (5.27), and (5.29).

The solution $y^{*}(t+s ; \psi, \zeta)$ is asymptotically orbitally stable if all eigenvalues of the operator (5.25) lie in the left complex half-plane and are unstable if $m$ roots, with multiplicities taken into account, belong to the right complex half-plane. In the latter case, the periodic solution has an m-dimensional unstable invariant periodic manifold.

Proof. Let us use the new time variable $\tau$ in (5.17)-(5.18) according to (5.19). As a result, we obtain a system of differential equations in space $l_{2} \otimes c$, which we write in the operator form

$$
\begin{aligned}
& \frac{d \rho}{d \tau}=\zeta^{2}\left(\gamma^{*}(\psi ; \zeta) \rho+R(\rho, \theta ; \psi, \zeta)+\zeta R^{\vee}(\rho, \theta, \tau ; \psi, \zeta)\right), \\
& \frac{d \theta}{d \tau}=\zeta^{2}\left(\delta^{*}(\psi ; \zeta)+\Theta(\rho, \theta ; \psi, \zeta)+\zeta \Theta^{\vee}(\rho, \theta, \tau ; \psi, \zeta)\right),
\end{aligned}
$$

The operators $\gamma^{*}(\cdot)=\left(\pi^{-1} \gamma_{1}^{*}(\cdot), \pi^{-1} \gamma_{-1}^{*}(\cdot), \ldots\right), \quad \delta^{*}(\cdot)=\left(\pi^{-1} \delta_{1}^{*}(\cdot), \pi^{-1} \delta_{-1}^{*}(\cdot), \ldots\right), \quad R(\cdot)=$ $=\left(\pi^{-1} R_{1}(\cdot), \pi^{-1} R_{-1}(\cdot), \ldots\right), \Theta(\cdot)=\left(\pi^{-1} \Theta_{1}(\cdot), \pi^{-1} \Theta_{-1}(\cdot), \ldots\right)$, and the operators $R^{\vee}(\cdot)=$ $=\left(R_{1}^{\vee}(\cdot), R_{-1}^{\vee}(\cdot), \ldots\right), \Theta^{\vee}(\cdot)=\left(\Theta_{1}^{\vee}(\cdot), \Theta_{-1}^{\vee}(\cdot), \ldots\right)$, are constructed according to functionals $R_{k}^{\vee}(\cdot)$ and $\Theta_{k}^{\vee}(\cdot)$, whose properties are determined by the properties of the functionals $R_{k}^{*}(\cdot)$, $\Theta_{k}^{*}(\cdot), T^{*}(\cdot), T(\cdot)$.

Denote by

$$
\bar{R}_{k}^{\vee}(\rho, \theta ; \psi, \zeta)=\frac{1}{2 \pi} \int_{0}^{2 \pi} R_{k}^{\vee}(\rho, \theta, \tau ; \psi, \zeta) d \tau, \quad \bar{\Theta}_{k}^{\vee}(\rho, \theta ; \psi, \zeta)=\frac{1}{2 \pi} \int_{0}^{2 \pi} \Theta_{k}^{\vee}(\rho, \theta, \tau ; \psi, \zeta) d \tau
$$

the mean of the function $R_{k}^{\vee}(\cdot)$ and $\Theta_{k}^{\vee}(\cdot)$ and consider in $E$ the system of nonlinear operator equations

$$
\begin{aligned}
& \gamma^{*}(\psi ; \zeta) \rho+R(\rho, \theta ; \psi, \zeta)+\zeta \bar{R}^{\vee}(\rho, \theta ; \psi, \zeta)=0 \\
& \delta^{*}(\psi ; \zeta) \rho+\Theta(\rho, \theta ; \psi, \zeta)+\zeta \bar{\Theta}^{\vee}(\rho, \theta ; \psi, \zeta)=0,
\end{aligned}
$$


with domain $E^{1}$, where the operators $\bar{R}^{\vee}(\cdot), \bar{\Theta}^{\vee}(\cdot)$ are defined according to (5.33). For $\zeta=0$ the system $(5.34)-(5.35)$ has the solution $\rho^{*}(\psi), \theta^{*}(\psi)$. In this case, the eigenvalues $\mu_{j}(\psi)$ of the operator (5.25), constructed from the partial derivatives matrix (5.24) corresponding to this solution, satisfy the condition $\left|\operatorname{Re} \mu_{j}(\psi)\right|>\mu_{0}>0$. Consequently, by the implicit function theorem for operator equations in a Banach space (see, for example, [12, pp. 304]) there is a $\zeta_{0}$ such that, when $0 \leqslant \zeta \leqslant \zeta_{0}$, the system of equations $(5.34)-(5.35)$ has the solution $\rho^{*}(\psi ; \zeta)$, $\theta^{*}(\psi ; \zeta)\left(\rho^{*}(\psi ; 0)=\rho^{*}(\psi), \theta^{*}(\psi ; 0)=\theta^{*}(\psi)\right)$ smoothly dependent on the parameter $\zeta$.

Linearizing (5.34) and (5.35) on the solution $\rho^{*}(\psi ; \zeta), \theta^{*}(\psi ; \zeta)$, we obtain an infinite matrix that is similar to the matrix (5.24) and turns into it when $\zeta=0$, and defines a linear operator

$$
B(\psi, \zeta) v \quad(v=(\rho, \theta), B(\psi, 0) v \equiv B(\psi) v): E^{1} \rightarrow E .
$$

Note that the eigenvalues $\mu_{j}(\psi, \zeta)\left(\mu_{j}(\psi, 0)=\mu_{j}(\psi)\right)$ of operator (5.36) for $0<\zeta<\zeta_{0}$ satisfy the condition $\left|\operatorname{Re} \mu_{j}(\psi, \zeta)\right|>\mu_{0}>0$.

We introduce the Banach spaces $C_{2 \pi}, C_{2 \pi}^{1}$ of, respectively, continuous and continuously differentiable $2 \pi$-periodic functions $f(\tau)(-\infty<\tau<\infty)$ with values in $E$ by defining the norms as $\|f\|_{C_{2 \pi}}=\sup _{\tau}\|f(\tau)\|_{E},\|f\|_{C_{2 \pi}^{1}}=\|f\|_{C_{2 \pi}}+\left\|f^{\prime}\right\|_{C_{2 \pi}}$.

Denote by $E_{+}(\psi, \zeta)$ and $E_{-}(\psi, \zeta)$ the root subspaces of the operator $(5.36)\left(\operatorname{dim} E_{+}(\cdot)=m\right)$, which correspond, respectively, to eigenvalues with positive and negative real parts, $E_{+}(\psi, \zeta) \oplus$ $\oplus E_{-}(\psi, \zeta)=E^{1}$. We denote the corresponding projections by $P_{+}(\psi, \zeta)$ and $P_{-}(\psi, \zeta)\left(P_{+}(\psi, \zeta)+\right.$ $\left.+P_{-}(\psi, \zeta)=I\right), P_{ \pm}(\psi, \zeta) E_{+}{ }^{1}=E_{ \pm}(\psi ; \zeta)$.

Consider in $E$ the linear differential equation

$$
\frac{d v}{d t}=B(\psi, \zeta) v
$$

Let $T(\tau ; \psi, \zeta)(\tau \geqslant 0)$ be a semigroup of bounded linear operators $T(\cdot): E^{1} \rightarrow E^{1}\left(T\left(\tau_{1}+\right.\right.$ $\left.\left.+\tau_{2} ; \cdot\right)=T\left(\tau_{1} ; \cdot\right) T\left(\tau_{2} ; \cdot\right)=T\left(\tau_{2} ; \cdot\right) T\left(\tau_{1} ; \cdot\right)\right)$ which defines the solutions (5.37) for $\tau \geqslant \tau_{0}$ with initial condition $v_{0}=v\left(\tau_{0}\right): v(\tau ; \psi, \zeta)=T(\tau ; \psi, \zeta) v_{0}$. We leave the previous notation for the continuous expansion $T(\cdot), P_{+}(\cdot), P_{-}(\cdot)$ on $E\left(E_{+}(\cdot) \oplus E_{-}(\cdot)=E\right)$.

We introduce the Green's operator defined on $E$ of the equation

$$
G(\tau ; \psi, \zeta)= \begin{cases}T_{+}(\tau ; \psi, \zeta)=T(\tau ; \psi, \zeta) P_{+}(\psi, \zeta), & t<0 \\ T_{-}(\tau ; \psi, \zeta)=T(\tau ; \psi, \zeta) P_{-}(\psi, \zeta), & t \geqslant 0 .\end{cases}
$$

In (5.38) the operator $T_{+}(\cdot)$ is finite-dimensional, and therefore it can be continued to the domain $\tau<0$ in a unique way. Also, the following estimates are valid:

$$
\left\|T_{+}(\tau ; \psi, \zeta)\right\|_{*} \leqslant k e^{\mu_{0} \tau}(\tau \leqslant 0),\left\|T_{-}(\tau ; \psi, \zeta)\right\|_{*} \leqslant k e^{-\mu_{0} \tau}(\tau \geqslant 0),
$$

where $\|T(\cdot)\|_{*}=\sup _{\|v\|_{E}=1}\|T(\cdot) v\|_{E}$.

Consider in $E$ the differential equation

$$
\frac{d v}{d \tau}=\zeta^{2} B(\psi, \zeta) v+f(\tau), f(\tau) \in C_{2 \pi}
$$

Equation (5.40) has the single $2 \pi$-periodic solution

$$
v(\tau ; \psi, \zeta))=\int_{-\infty}^{\infty} G\left(\zeta^{2}\left(\tau-\tau_{1}\right)(\psi ; \zeta)\right) f\left(\tau_{1}\right) d \tau_{1}, \quad\left(v(\tau, \psi, \zeta) \in C_{2 \pi}^{1}\right)
$$


and, by virtue of (5.39),

$$
\begin{gathered}
\|v(\tau ; \psi, \zeta)\|_{C_{2 \pi}^{1}} \leqslant\left\|\int_{-\infty}^{\tau} T_{-}\left(\zeta^{2}\left(\tau-\tau_{1}\right) ; \psi, \zeta\right) f\left(\tau_{1}\right) d \tau_{1}\right\|_{C_{2 \pi}^{1}}+\left\|\int_{\tau}^{\infty} T_{+}\left(\zeta^{2}\left(\tau-\tau_{1}\right) ; \psi, \zeta\right) f\left(\tau_{1}\right) d \tau_{1}\right\|_{C_{2 \pi}^{1}} \leqslant \\
\zeta^{-2} 2 k / \mu_{0}\left\|f\left(\tau_{1}\right)\right\|_{C_{2 \pi}} .
\end{gathered}
$$

The formula (5.41) is checked by a direct substitution into Eq. (5.40). The stability of the $2 \pi$-periodic solution (5.41) is determined by the location of the eigenvalues of the operator (5.36). For $m=0$ the solution (5.41) is asymptotically stable, and for $m>0$ the solution (5.41) has an $m$-dimensional invariant unstable manifold.

We show that the system of equations (5.31)-(5.32) has a $2 \pi$-periodic solution of the form

$$
\begin{aligned}
& v^{*}(\tau ; \psi, \zeta)=v^{*}(\psi, \zeta)+v_{1}^{*}(\tau ; \psi, \zeta)= \\
& \quad=\left(\rho^{*}(\psi, \zeta), \theta^{*}(\psi, \zeta)\right)+\left(\rho_{1}^{*}(\tau ; \psi, \zeta), \theta_{1}^{*}(\tau ; \psi, \zeta)\right), \quad v_{1}^{*}(\tau ; \psi, \zeta) \in C_{2 \pi}^{1}
\end{aligned}
$$

Assuming $v_{1}(\tau)=v_{1}(\tau ; \cdot)$ in $(5.42)$ as the required function (we omit the sign ${ }^{*}$ ), we substitute into (5.31), (5.32). As a result, we obtain an equation for determining $v_{1}(\tau)$ of the following form

$$
\frac{d v_{1}}{d \tau}=\zeta^{2}\left(B(\psi, \zeta) v_{1}+F\left(v_{1} ; \psi, \zeta\right)+\zeta F^{\vee}\left(\tau, v_{1} ; \psi, \zeta\right)+\zeta f^{*}(\tau, \psi ; \zeta)\right),
$$

in which the function $f^{*}(\tau ; \psi, \zeta) \in C_{2 \pi}$ and the operators $F(\cdot), F^{\vee}(\cdot)$, smoothly depending on the parameters and variables, satisfy the conditions $\left\|F\left(v_{1} ; \psi, \zeta\right)\right\|_{E}=O\left(\left\|v_{1}\right\|_{E^{1}}^{2}\right), F^{\vee}(\tau, 0 ; \psi, \zeta) \equiv 0$, with the averages $\bar{f}^{*}(\psi ; \zeta) \equiv 0$ and $\bar{F}^{\vee}\left(v_{1} ; \psi, \zeta\right)=0$.

The problem of the existence of a solution $v_{1}(\tau) \in C_{2 \pi}^{1}$ of Eq. (5.44) based on (5.40) and (5.41) is equivalent to the solvability of the following operator equation:

$$
v_{1}(\tau)=\zeta^{2} \int_{-\infty}^{\infty} G\left(\zeta^{2}\left(\tau-\tau_{1}\right)\left(F\left(v_{1}\left(\tau_{1}\right) ; \psi, \zeta\right)+\zeta F^{\vee}\left(\tau_{1}, v_{1}\left(\tau_{1}\right) ; \psi, \zeta\right)+\zeta f^{*}\left(\tau_{1} ; \psi, \zeta\right)\right) d \tau_{1}\right.
$$

in space $C_{2 \pi}^{1}$. Based on (5.42), we can apply the implicit function theorem for operator equations in a Banach space [12, pp. 304] to the operator equation (5.45). As a result, we obtain the solution $v_{1}^{*}(\tau ; \psi, \zeta)(v(\tau ; \psi, 0) \equiv 0)$ of Eq. (5.45), which smoothly depends on the incoming parameters. Performing inverse substitutions, we have a periodic solution $y^{*}(t+s ; \psi, \zeta)$. Formula (5.30) was obtained using (4.13) and (4.16) (with $s=0),(5.19)$ by taking into account only $\left(\rho^{*}(\psi), \theta^{*}(\psi)\right)$ the "main part" of the solution (5.43). The stability of the periodic solution $y^{*}(t+s ; \psi, \zeta)$ is determined by the stability of the periodic solution of Eq. (5.40). The theorem is proved.

\section{Numerical investigation of the normal form of Eq. (2.7)}

Here are some of the results of numerical analysis of stable equilibrium states $\left(\rho^{*}(\psi), \theta^{*}(\psi)\right)$ of the system of differential equations (5.20)-(5.21) (for $\zeta=0$ ), which are solutions of the system of nonlinear algebraic equations (5.22)-(5.23) and define, according to (5.26), (5.29)(5.30), stable periodic solutions of equation (2.1), which bifurcate from an equilibrium state $x_{*}\left(\mu_{*}\left(c_{*}\right), c_{*}\right)$ for the case $c_{*}=\pi / 3$. In this case, $x_{*}\left(\mu_{*}\left(c_{*}\right), c_{*}\right) \approx-1.33, \mu_{*}\left(c_{*}\right) \approx 1.56$. 


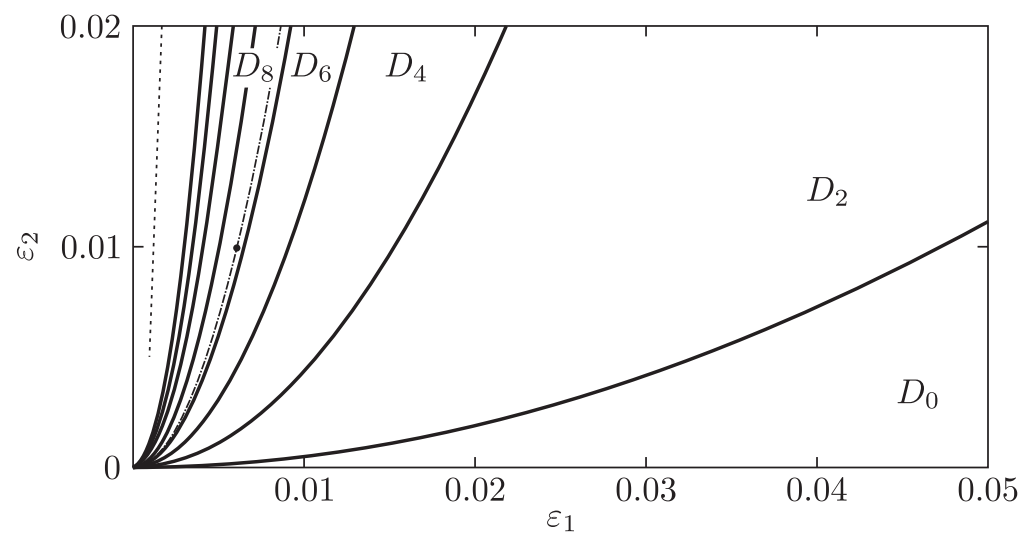

Fig. 3. Picture of $D-$ partitions.

Figure 3 presents a picture of $D$-partitions [13] of the parameter plane $\left(\varepsilon_{1}, \varepsilon_{2}\right)$. It shows the curves on which Eq. (2.10) has roots lying on the imaginary axis. These curves are the boundaries of domains of $D$-partitions. For values of $\varepsilon$ from the area $D_{0}$ all roots of the characteristic equation (2.10) lie in the left complex half-plane, for $\varepsilon \in D_{2}$ one pair of complex conjugate roots is in the right complex half-plane, and the remaining pairs are in the left one, for $\varepsilon \in D_{4}$ two pairs of complex-conjugate roots are in the right complex half-plane, and the remaining pairs are in the left one, and so on. The dotted line denotes the boundaries of subsequent regions of $D$-partitions, which are similar to those described above. We note that the reduced picture of $D$-partitions of the parameter plane $\left(\varepsilon_{1}, \varepsilon_{2}\right)$ can be easily mapped, according to $(2.6)$, to the parameter plane $\left(\varepsilon_{1}, \mu\right)$.

Fix the values of the parameters $\psi=1.51$ in (5.16). This value of $\psi$ in Fig. 3 corresponds to a curve in the domain $D_{8}$, marked by points. For this value of $\psi$, stable equilibrium states of the system of differential equations (5.20)-(5.21) (for $\zeta=0$ ) were defined numerically. Calculations were carried out according to the following scheme. A sequence of truncated finite-dimensional systems (5.20)-(5.21) (with $\zeta=0$ ), where $k=1,3, \ldots, N$ was considered. The finite-dimensional system of differential equations was numerically integrated under various initial conditions. For numerical integration we used the software complex [14], which allowed us to simultaneously build a large number of integral trajectories (with an automatic choice of the integration step) for initial conditions randomly "abandoned", for example, according to the uniform law. There was a stable state of equilibrium $\left(\rho_{N}^{*}(\psi), \theta_{N}^{*}(\psi)\right)$. This equilibrium state was refined in the system of equations (5.20)-(5.21) (for $\zeta=0$ ) for $k=1,3, \ldots, N+1$ etc. until $\| \rho_{N}^{*}(\psi)-$ $-\rho_{N+1}^{*}(\psi)\left\|_{l_{2}^{1}} /\right\| \rho_{N}^{*}(\psi) \|_{l_{2}^{1}}<\Delta\left(\Delta=10^{-2}\right)$.

For the specified value of $\psi$ five stable equilibrium states of the system of differential equations (5.20)-(5.21) (for $\zeta=0$ ) were identified. There were no other stable equilibrium states. For each of the found equilibrium states a periodic solution of Eq. (2.1) was constructed according to $(2.4),(5.26),(5.29)-(5.30)$. Figure 4 shows for $\zeta=0.1$ (in Fig. 3, a bold point in area $D_{8}$ ) graphs of these solutions (the thick line) and their projections onto the plane $(x(t), x(t-1))$. The figures also show graphs of the solutions of Eq. (2.1), which were obtained by its numerical integration by the Euler method with constant step selection, with the same parameter values. The integration step was reduced until a complete stabilization of the computational results was achieved. The periodic functions (solutions) obtained in accordance with (2.4), (5.26), (5.29)-(5.30) were taken as initial functions. 

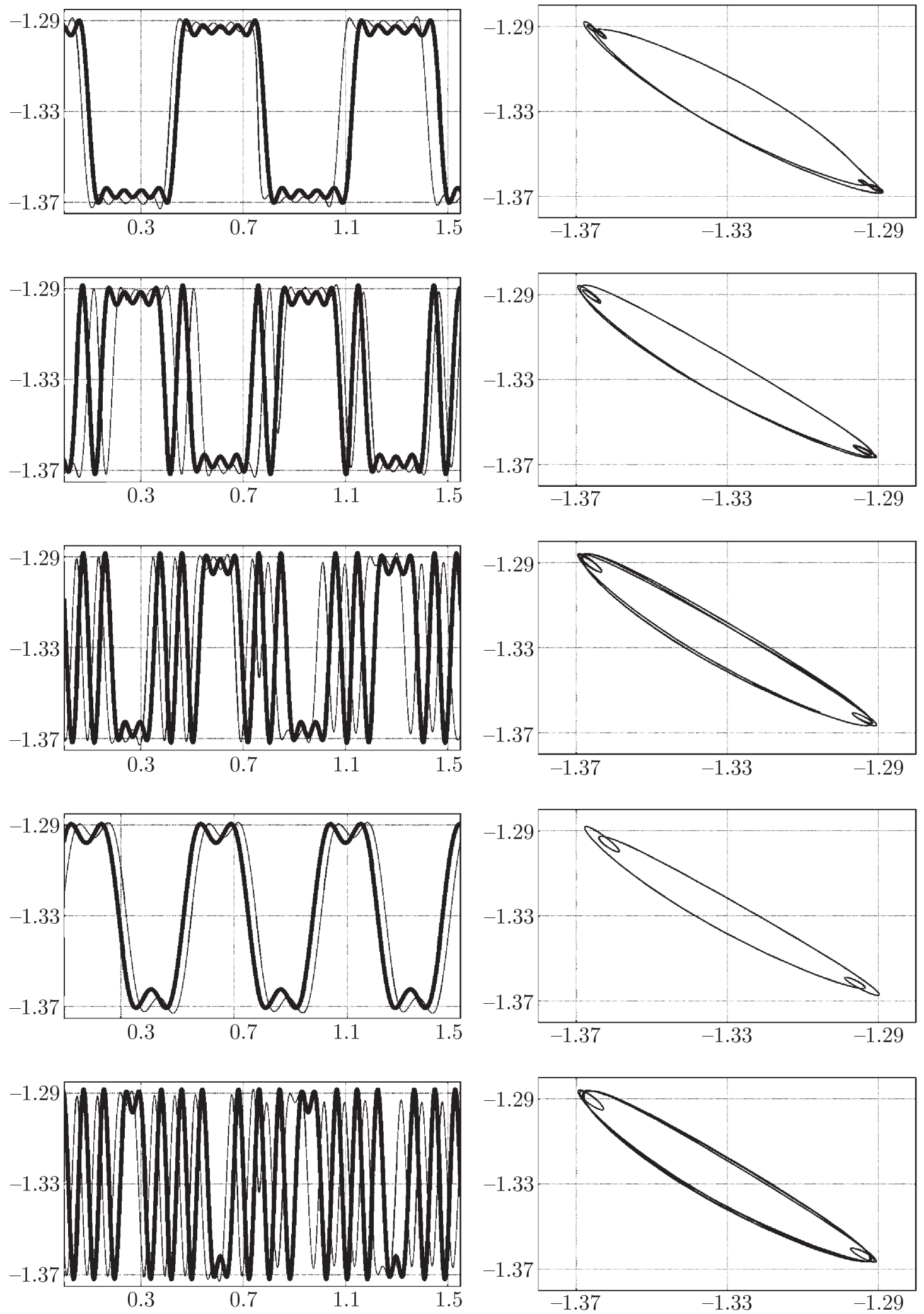

Fig. 4. Periodic solutions of Eq. (2.1) and the projection of phase portraits onto the plane $(x(t), x(t-1))$ for $\zeta=0.1, \psi=1.51$. 
Thus, for the selected value of the parameter $\psi$ with changing parameter $\zeta>0$ in Eq. (2.1), in which $\varepsilon_{1}$ and $\mu$ vary according to (2.6) and (5.16), a simultaneous bifurcation from the state (2.4) of five periodic solutions is observed. Such a phenomenon is called a bifurcation of multistability.

For other values of the parameter $\psi$, the system of equations (5.20)-(5.21) (for $\zeta=0$ ) also has a large number of stable equilibrium states to which the periodic solutions of Eq. (2.1) correspond. For example, for $\psi=1.55$, seven stable equilibrium states were identified. The number of equilibrium states increases with increasing $\psi$.

Now fix the value of the parameter $\psi=1.51$ and increase $\zeta$. In Fig. 3, this case is represented by the dotted line in region $D_{8}$. Let us consider how the resulting periodic solutions will change as $\zeta$ changes. As the parameter $\zeta$ increases, every periodic solution turns into irregular (chaotic) oscillations through a series of period doubling bifurcations. The maximum characteristic exponent was calculated for chaotic oscillations and turned out to be positive. In this case, there exists a range of values of the parameter $\zeta$ for which there are simultaneously five different chaotic oscillations (attractors). This phenomenon can be called chaotic multistability. For numerical integration of Eq. (2.1) and calculation of the maximum characteristic exponent, the software complex [14] was used. Figure 5 shows the projections of phase portraits of periodic solutions to the plane $(x(t), x(t-1))$ corresponding to the transition of the first two periodic solutions presented in Fig. 4 from regular oscillations to chaotic values for $\zeta=0.2,0.4,0.8$.
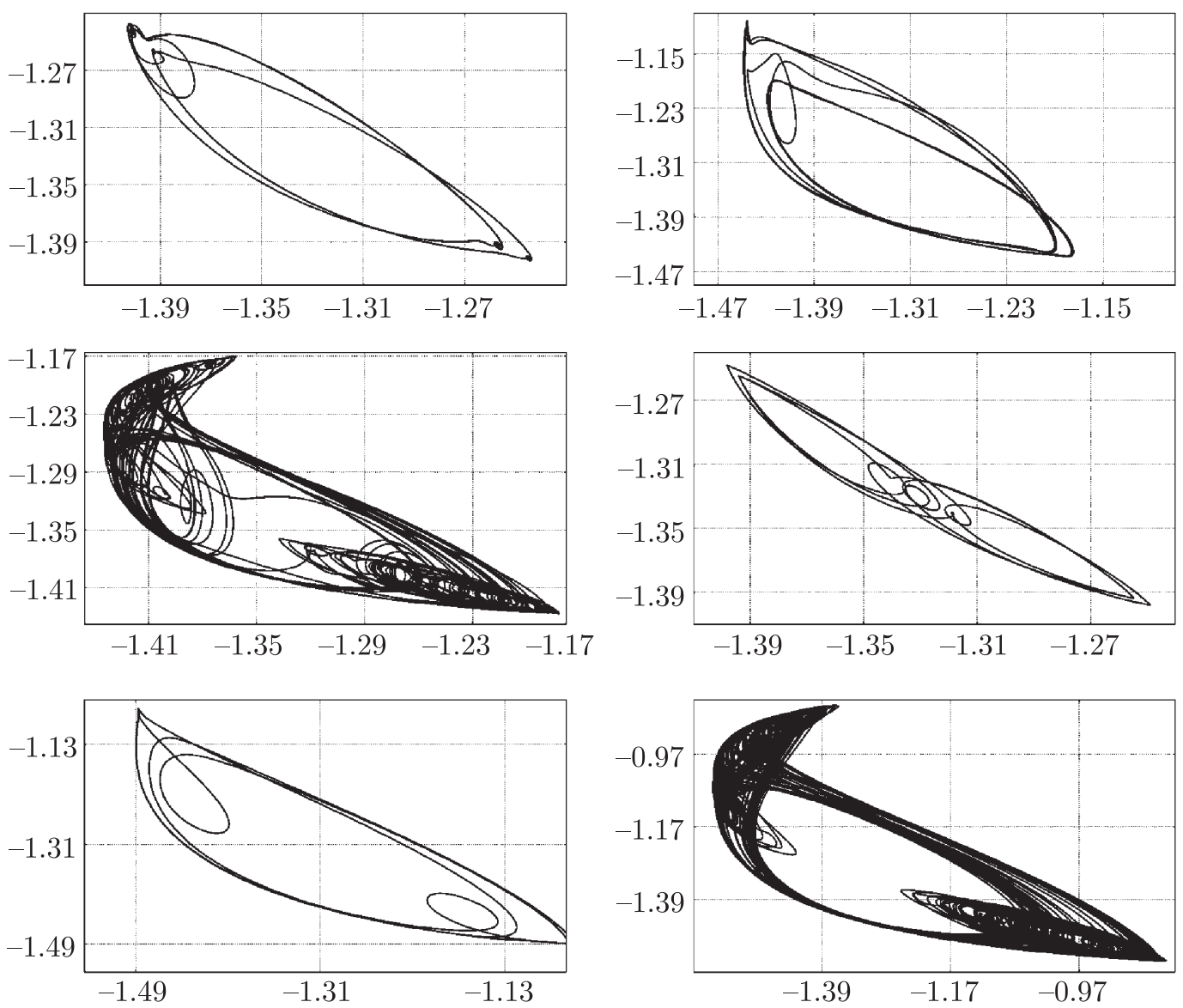

Fig. 5. Projections of phase portraits of the first and second periodic solutions of Eq. (2.1) onto the plane $(x(t), x(t-1))$ for the values $\psi=1.51, \zeta=0.2,0.4,0.8$. 


\section{References}

[1] Ikeda, K., Multiple-Valued Stationary State and Its Instability of the Transmitted Light a Ring Cavity System, Opt. Commun., 1979, vol. 30, no. 2, pp. 257-261.

[2] Ikeda, K., Daido, H., and Akimoto, O., Optical Turbulence: Chaotic Behavior of Transmitted Light from a Ring Cavity, Phys. Rev. Lett., 1980, vol.45, no. 9, pp. 709-712.

[3] Ikeda, K. and Matsumoto, K., High-Dimensional Chaotic Behavior in System with Time-Delayed Feedback, Phys. D, 1987, vol. 29, nos. 1-2, pp. 223-235.

[4] Sprott, J.C., Elegant Chaos: Algebraically Simple Chaotic Flows, Singapore: World Sci., 2010.

[5] Ponomarenko, V. I. and Prokhorov, M. D., Recovering Parameters of the Ikeda Equation from Noisy Time Series, Tech. Phys. Lett., 2005, vol.31, no.3, pp. 252-254; see also: Pis'ma Zh. Tekh. Fiz., 2005, vol.31, no. 6, pp. 73-78.

[6] Larger, L., Goedgebuer, J.-P., and Udaltsov, V., Ikeda-Based Nonlinear Delayed Dynamics for Application to Secure Optical Transmission Systems Using Chaos, C. R. Math. Acad. Sci. Paris, 2004, vol. 5, no. 6, pp. 669-681.

[7] Kubyshkin, E.P. and Nazarov, A. Yu., Analysis of Oscillatory Solutions of a Nonlinear Singularly Perturbed Differential-Difference Equation, Vestn. Nizhegorodsk. Univ., 2010, vol.5, no.2, pp. 118-125 (Russian).

[8] Kubyshkin, E. P. and Moriakova, A. R., Analysis of Bifurcations of Periodic Solutions of Ikeda Equation, Nonlinear Phenomena in Complex Systems, 2017, vol. 20, no. 1, pp. 40-49.

[9] Bellman, R. and Cooke, K. L., Differential-Difference Equations, New York: Acad. Press, 1963.

[10] Krein, S. G., Linear Differential Equations in Banach Space, Providence, R.I.: AMS, 1972.

[11] Shimanov, S. N., On the Vibration Theory of Quasilinear Systems with Lag, J. Appl. Math. Mech., 1959, vol. 23, no. 5, pp.1198-1208; see also: Prikl. Mat. Mekh., 1959, vol. 23, no. 5, pp. 836-844.

[12] Krasnosel'skii, M. A., Vainikko, G. M., Zabreyko, P. P., Ruticki, Ya. B., and Stetsenko, V. Ya., Approximate Solution of Operator Equation, Groningen: Wolters-Noordhoff, 1972.

[13] Neimark, Yu. I., D-Partitions of the Space of Quasipolynomials (to the Stability of Linearized Distributed Systems), Prikl. Mat. Mekh., 1949, vol.13, no.4, pp. 349-380 (Russian).

[14] Glyzin, D.S., The Program Package for the Analysis of Dynamic Systems "Tracer", Appl. no. 2008610548 dated Feb 14, 2008, Certificate of State Registration of the Computer Program no. 2008611464, Registered in the Register of Computer Programs 24.03.2008 (Russian). 